\title{
An Automatic Zone Detection System for Safe Landing of UAVs
}

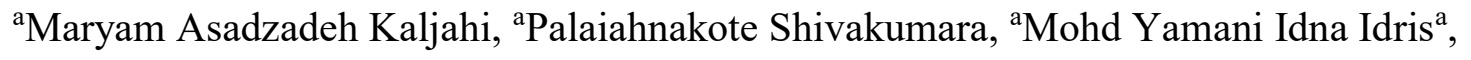 \\ ${ }^{b}$ Mohammad Hossein Anisi ${ }^{\mathrm{a}}$, ${ }^{\mathrm{c}}$ Tong Lu, ${ }^{\mathrm{d}}$ Michael Blumenstein and ${ }^{\mathrm{a}}$ Noorzaily Mohamed Noor \\ ${ }^{a}$ Faculty of Computer Science and Information Technology, University of Malaya, Kuala Lumpur, Malaysia. \\ ${ }^{\mathrm{b} S}$ School of Computer Science and Electronic Engineering, University of Essex, CO4 3SQ Colchester, UK. \\ ${ }^{\mathrm{c}}$ National Key Lab for Novel Software Technology, Nanjing University, Nanjing, China. \\ ${ }^{d}$ Faculty of Engineering and Information Technology, University of Technology Sydney, Sydney, Australia \\ asadzadeh@um.edu.my, shiva@um.edu.my,yamani@um.edu.my,m.anisi@essex.ac.uk, lutong@nju.edu.cn \\ michael.blumenstein@uts.edu.au, zaily@um.edu.my
}

\begin{abstract}
As the demand increases for the use Unmanned Aerial Vehicles (UAVs) to monitor natural disasters, protecting territories, spraying, vigilance in urban areas, etc., detecting safe landing zones becomes a new area that has gained interest. This paper presents an intelligent system for detecting regions to navigate a UAV when it requires an emergency landing due to technical causes. The proposed system explores the fact that safe regions in images have flat surfaces, which are extracted using the Gabor Transform. This results in images of different orientations. The proposed system then performs histogram operations on different Gabor-oriented images to select pixels that contribute to the highest peak, as Candidate Pixels (CP), for the respective Gabor-oriented images. Next, to group candidate pixels as one region, we explore Markov Chain Codes (MCCs), which estimate the probability of pixels being classified as candidates with neighboring pixels. This process results in Candidate Regions (CRs) detection. For each image of the respective Gabor orientation, including CRs, the proposed system finds a candidate region that has the highest area and considers it as a reference. We then estimate the degree of similarity between the reference $\mathrm{CR}$ with corresponding CRs in the respective Gabor-oriented images using a Chi square distance measure. Furthermore, the proposed system chooses the CR which gives the highest similarity to the reference CR to fuse with that reference, which results in the establishment of safe landing zones for the UAV. Experimental results on images from different situations for safe landing detection show that the proposed system outperforms the existing systems. Furthermore, experimental results on relative success rates for different emergency conditions of UAVs show that the proposed intelligent system is effective and useful compared to the existing UAV safe landing systems.
\end{abstract}

Keywords: UAV, Disaster management, Safe landing zone, Gabor transform, Markov Chain Code, Safe landing detection. 


\section{Introduction}

Due to the large number of unexpected natural disasters and social crimes, the use of Unmanned Aerial Vehicles (UAVs) is in demand. This is because of several key features of UAVs, such as low cost, high mobility, flexibility, safety and customizability (Kaff et al., 2018; Patterson et al., 2010). The observation and monitoring of the above-mentioned situations have become much easier and faster because UAVs can reach an area of interest in a short period of time (Fan et al., 2018). UAVs are commonly known as a drone, which describes the aircraft platform without a human pilot onboard (Huang et al., 2016). More than 70\% of UAVs are used for military purposes, such as battlefield reconnaissance and surveillance, positioning, fire and damage assessment. In addition, the recent use of UAVs for civilian applications has increased, especially for border patrol, environmental exploration, aerial photography and resource exploration, disaster monitoring, capturing views of auditoriums where live sports are telecast, welcoming very important persons by deploying flowers from the sky, and spying during attacks. It is true that usually outdoor UAVs perform tasks according to the instructions given by planned paths using a satellite-based system, such as a global positioning system (GPS). However, there are situations where the GPS may not function well due to adverse environmental factors (Patterson et al., 2010, Pulido et al., 2017), which results in GPS-denial. As a result, one cannot expect the GPS system to work well in all situations, especially under poor space weather conditions, which affect the signal strength and reliability. Further details on this issue can be found in (Lee et al., 2017). On top of this, sometimes, static and dynamic obstacles, engine failures, and security attacks may create emergencies as shown in Fig. 1. In order to find a solution to these sensitive issues, one needs an alternative way of finding locations with the help of image processing-based expert systems for safe landing. Note: in this work, we consider small or medium UAVs for experimentation. For

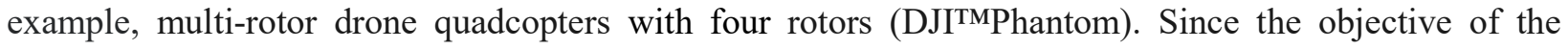
proposed work is to find a safe landing zone when a UAV is facing emergency-situations, the proposed work does not need a clear runway for landing, rather it only finds a safe zone for landing.

There are existing systems to handle the above situations, which follow the steps shown in Fig. 2. During emergencies, as mentioned in Fig. 2, an automatic system called a fault detection unit sends an alert message to another unit for exploring safe zones. To identify a safe zone for landing, existing image processingbased systems focus on segmentation of regions of interest (zone detection), such as greenery, forest, river and mountain. Then the systems classify the segmented regions such as river, mountain, greenery, forest

and road to find safe and flat zones; this can be considered as zone verification for the detected zones. In Fig. 2, case-1, case-2 and case-3 refer to emergencies created due to communication failures from ground stations, e.g. GPS failures and software-hardware/energy failures, respectively (Patterson et al., 2014). 


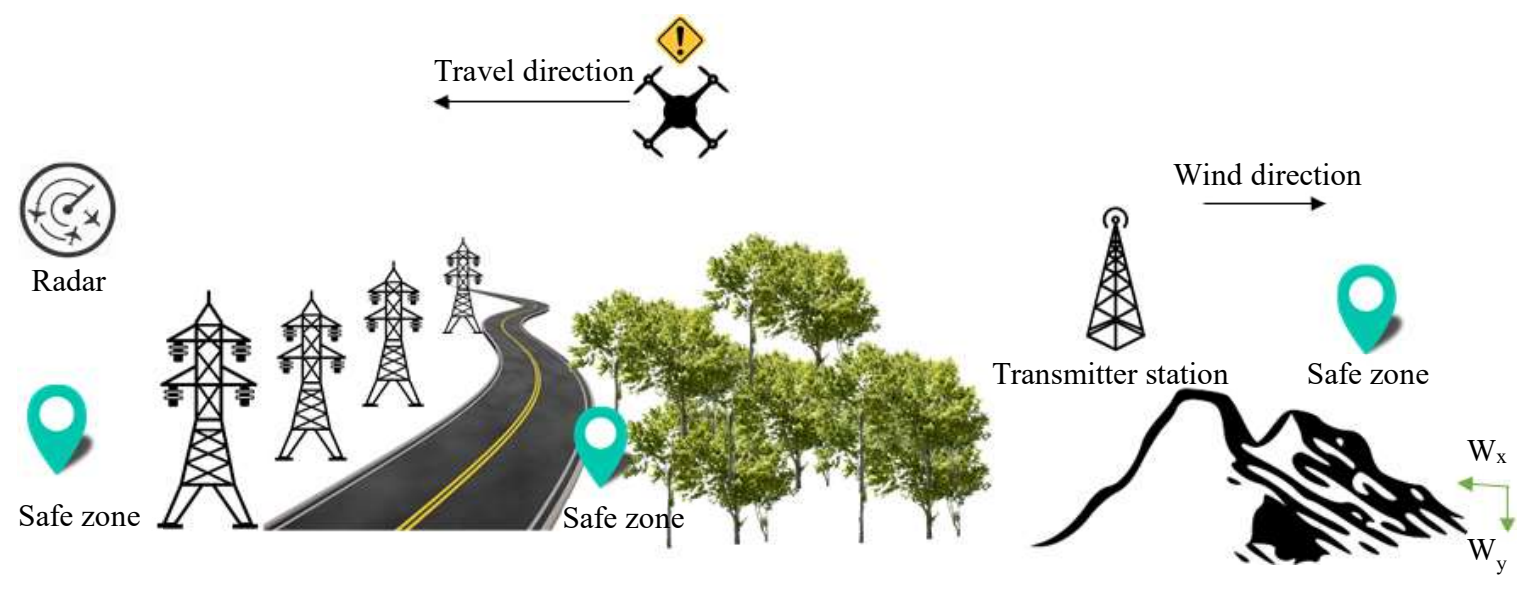

Fig .1. Examples of emergencies whilst a UAV is flying.

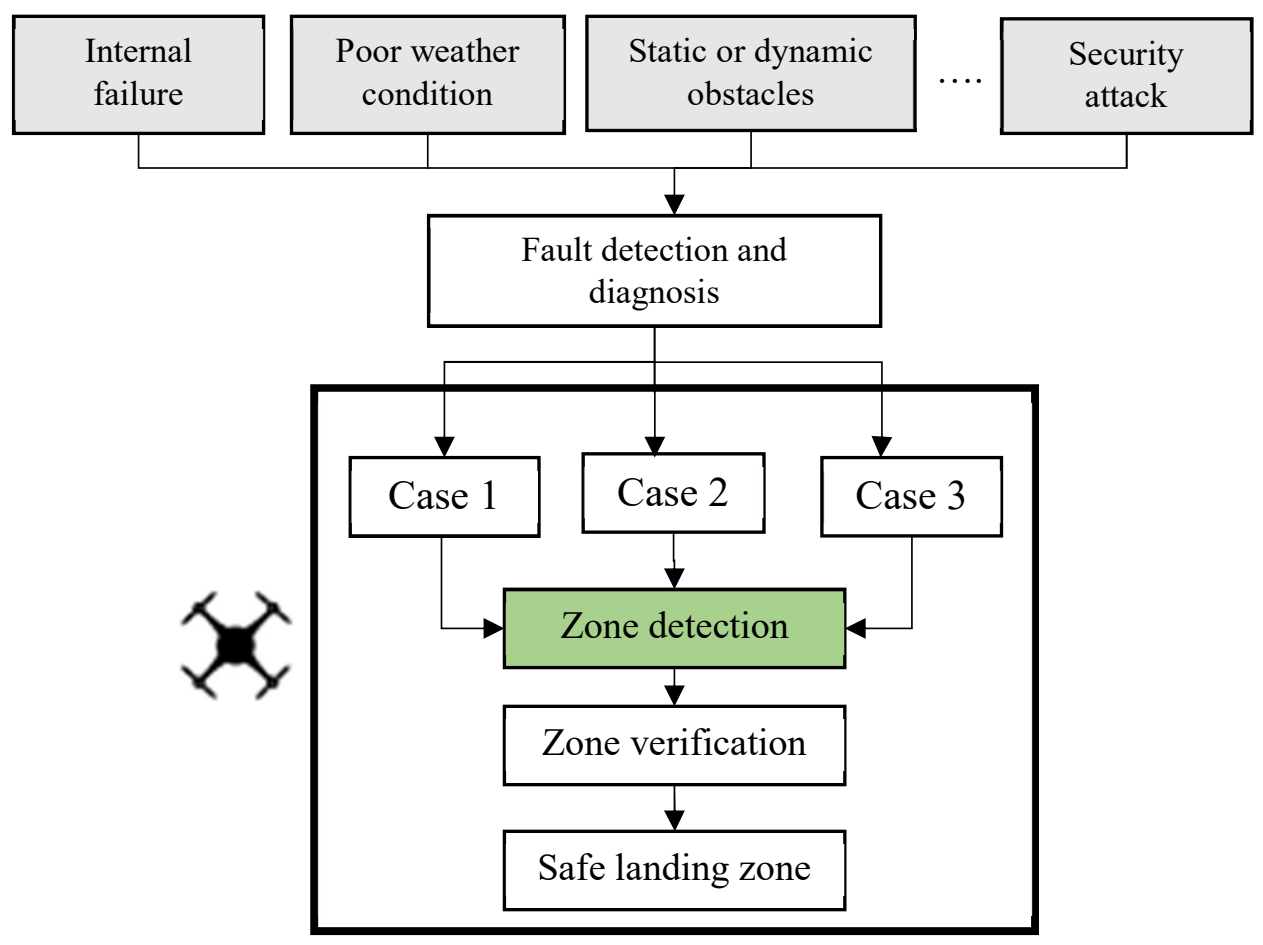

Fig .2. General framework for safe landing of UAVs

It is noted that defining regions of interest and the classification of particular regions (zones) is reliable when we have a limited number of regions. However, it is not necessarily true for all situations, where we can anticipate unexpected regions such as buildings, towers, small plants, and flat regions with cars or other objects. In addition, one can say that shapes and the nature of regions could be infinite. It is evident from the sample results of one of the existing systems (Patterson et al., 2014), as shown in Fig. 3. In Fig. 3, it can be seen that for different input images in (a), the proposed system detects flat regions (white pixels) which 
can be used for safe landing for all the four situations, while the existing system fails in the case of the fourth image and does not detect accurately the first three images shown in Fig. 3(c). The main reason is that the existing system has inherent limitations of segmentation and classification. Therefore, it is necessary to develop a system which can detect zones irrespective of the content of the regions. Hence, in this work, we propose an intelligent system, which works based on the fact that Gabor orientation responses are low for flat or homogeneous regions, whilst they are high for the other regions where some obstacles exist. The main advantage of this idea is that it does not require either region-specific features for segmentation or classification as in existing methods. This is the key contribution of the proposed system, when compared to the state-of-the-art systems.
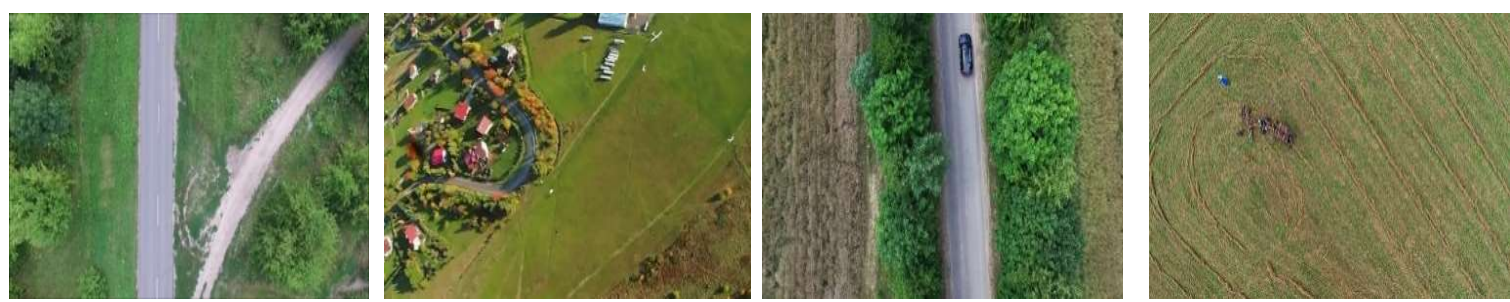

(a) Input images captured by UAVs of different regions.
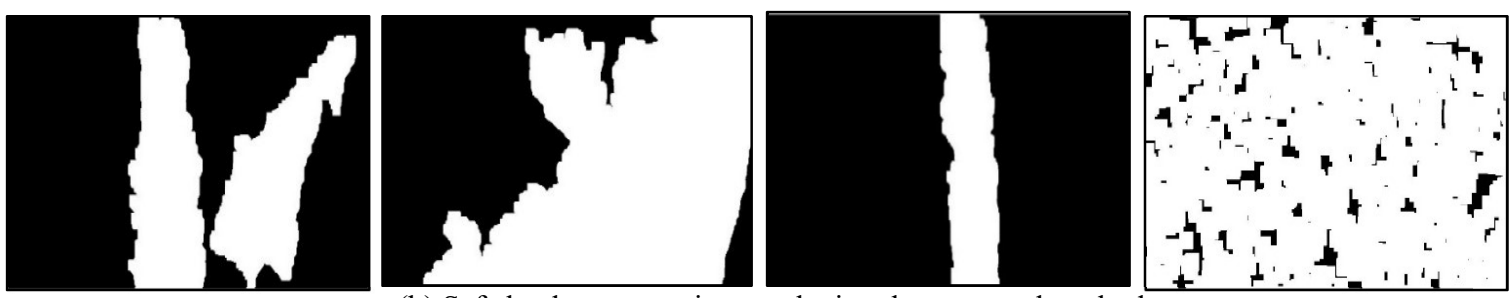

(b) Safe land segmentation employing the proposed method
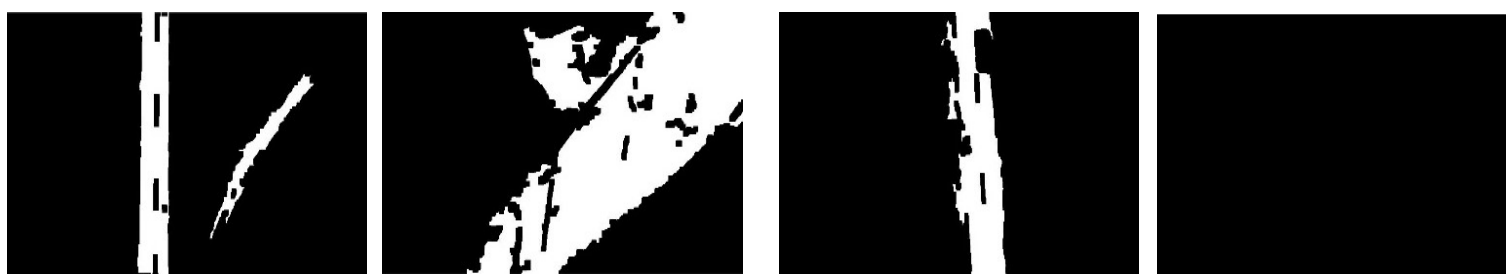

(c) Safe land segmentation in an existing method (Patterson et al., 2014)

Fig. 3. Challenges of safe land segmentation.

\section{Related Work}

Since the proposed work focuses on the segmentation of flat regions which can be used for the safe landing of UAVs, we review the methods that perform zone segmentation from different images captured by UAVs, rather than the general methods (Ghamisi et al., 2012) that segment regions from scene images and other complex images. This is due to the fact that most general segmentation methods usually propose texture, color and edge-based features to define regions of interest (Ghamisi et al., 2012). In addition, the methods make use of the shapes of objects for achieving better results. However, flat regions, which are required for 
safe landing of UAVs, cannot be defined by texture or color because they can have any texture, color, or mixed with different textures and colors. Besides, one cannot expect objects with specific shapes in the case of flat regions. Therefore, we review the methods that detect flat regions for safe landing as in the proposed work.

Huang et al. (2016) proposed geological segmentation for UAV aerial images using shape-based LSM with dominant color. The method uses fuzzy c-means clustering for classification based on color information. It finds dominant colors and then estimates a similar distribution map. Furthermore, a shape-based level set method is proposed to segment regions. The method works well for images where dominant colors represent flat regions. However, this is not true for many cases.

Hinzmann et al. (2018) proposed prior knowledge-free visual landing site detection for autonomous planes. The method explores texture and geometric shapes without using any prior knowledge about the environment. The method considers hazards within landing regions such as terrain roughness and slope, surrounding obstacles, which cause local wind fields. However, it is our hypothesis that flat regions may have arbitrary shapes and colors.

Aydin \& Kugu (2016) proposed safe landing site detection using shuttle radar topography mission (SRTM) maps for UAVs. Initially, the method segments regions of interest called blobs, which are analyzed for landing zone detection. Since the success of the method depends on the success of segmentation, therefore, the method may not perform well for complex images.

Lange et al. (2008) proposed autonomous landing for a multirotor UAV using vision. The method explores adaptive binarization for segmenting foreground and background information. Then the components in ten binary images are defined as connected components to study their shapes. Small components between rings are removed using some threshold values. However, this method is limited to particular types of vision from a UAV, but not general land zone detection in different situations.

Patterson et al. (2014) proposed timely autonomous identification of UAV safe landing zones. The method combines ordnance surveys and Canny edge images of the input image for potential region detection. The method estimates safety scores based on properties of regions to eliminate obstacles in an image. Further, the method proposes fuzzy-based features for the classification of regions. This helps the method to classify regions with paths, trees, rivers, etc., and homogenous zones are determined based on the roughness of the regions. The method is limited to regions where there are no complex backgrounds because a Canny edge operator is sensitive to it. In addition, sometimes, such a region may contain unexpected areas and surfaces for classification. 
Scherer et al. (2012) proposed autonomous landing at unprepared sites by full-scale helicopters. The method focusses on the factors of environmental issues, such as terrain, skid intersections, rotor, tail clearance, wind direction, clear approaches, abort paths and ground paths. In order to estimate the previously mentioned parameters, the method considers the direction of the helicopter, geometrical properties of the land, etc. In other words, the method studies reflections of locations for safe landing without analyzing the surface of the ground. This is suitable when all constituent parts and units are in good condition. However, the target of the proposed work is only for emergency situations where we can expect technical failures in the system. Garg et al. (2015) proposed terrain-based landing site selection and path planning for fixed-wing UAVs. This method uses terrain information from digital elevation maps to find a list of potential landing sites. It selects a site from a list of potential sites, and then finds a path between the selected site and the UAV. Basically, the method studies geometrical properties of the chosen sites from the elevation map to confirm appropriate landing locations. This is applicable when there are no technical failures, but not for the situations considered in this work.

Aziz et al. (2016) proposed a UAV emergency landing site identification system using machine vision. The method explores Canny edge information and a histogram of the ground images for finding safe landing areas. In addition, the method extracts statistical features from the ground to verify the detected safe landing sites. However, it is not clear how the method works for complex background images because Canny is sensitive to complex backgrounds. In addition, histogram operations and thresholding may not work well for different situations.

Li (2013) proposed a software scheme for UAV safe landing area discovery. The method explores a similarity-based texture area for finding possible landing areas. Then the features are passed to an SVM classifier for confirming landing areas. As discussed above, textures alone are not sufficient to define flat regions or landing regions because they can have any texture and sometimes no texture. In addition, the area can contain plants of the same size and surface, which appear like textures. Therefore, the method has inherent limitations when we compare it to the work considered here.

From the above review of the existing systems on safe zone detection, it is noted that most systems use texture, edge and other geometrical features for the detection of suitable landing sites. In addition, the methods focus on the classification of segmented regions based on the nature of surfaces for verification. In other words, the existing systems need prior knowledge of regions to define the features. However, it is correct that defining features for unexpected regions is challenging, and hence the existing systems are appropriate for a limited number of regions. Therefore, there is a need for developing generalized systems for safe zone detection without prior knowledge of the land and terrain. Hence, in this work, we propose a new expert system for safe zone detection based on the responses of Gabor filters. The proposed system 
works based on the fact that UAVs can land on any region which has an appropriate flat surface. It is true that for flat surfaces where we can see plain and homogenous areas, one can expect uniform responses from Gabor filters, and arbitrary responses for the other areas. As a result, the proposed system does not require any specific features to define flat surfaces and for classification to find safe locations for landing. This is the key contribution in the present work, which is different from the existing systems.

\section{Proposed System}

As technology advances, we can see significant improvements in UAV landing. As a result, UAVs require flat areas irrespective of color, size, and even to some extent poor weather conditions. With this notion, we explore the fact that landing zones should be flat or homogenous/plain areas for safe landing. For example, it could be a terrace, roof, ground, grass region, road, field, etc. To extract such an observation, motivated by the method of (Saxena et al., 2018) where the Gabor response of different orientations is proposed for finger print identification based on the fact that Gabor filters give low degrees of response for homogeneous areas and a high degree of variances for non-flat ones, we explore Gabor filters of different orientations for safe zone detection, which reflect flat regions. For input images captured by UAVs, the proposed system first obtains Gabor orientation responses of eight directions. The number of orientations (eight) was determined based on experimental analysis, and it is valid because those directions generally provide prominent information about objects in images. It is expected that flat regions provide uniform Gabor responses for their respective directions, and the proposed system performs histogram operations over Gabor response images. Then, it finds the responses that contribute to the highest peak in the histogram as candidate pixels of the respective eight response images. As noted, Markov Chain Codes (MCCs) estimate the probability of using previous states to predict new states (Mulatu, 2017). Inspired by this property, we estimated the probability of using eight neighbors from each pixel in the candidate pixel images of the respective eight responses, which predicts whether the center pixels are light or dark. This process results in clusters of pixels that share the same property of the center pixels, which are called Candidate Regions (CR).

One can expect each response may contain several CRs due to variations in the Gabor orientations and the nature of images. Therefore, the proposed system finds CRs, which give the highest areas compared to their corresponding CRs of all the response images; these are considered as reference CRs. It is noted that CRs in the response images have different areas. As a result, it is hard to ensure that the reference CR represents expected homogenous safe landing zones. To deal with this issue, for each $\mathrm{CR}$ in all the responses, the proposed system performs histogram operations over probability values given by the MCCs for the pixels in the CR (as discussed above). It then estimates degrees of similarity between the histogram distributions through Chi square distance measures. Motivated by the method of (Ghosh et al., 2012) where Chi square 
has been used for estimating degrees of similarity between two histograms of the regions for object detection, we use the same in our work. The proposed system finds the most similar CR to the reference $\mathrm{CR}$ in each response image. This process eliminates non-CR regions as well as providing correct $\mathrm{CR}$ regions. With this advantage, the proposed system fuses the reference CR with the most similar region out of other regions in the response images. This results in the expected homogenous regions for safe landing of UAVs. The workflow of the proposed method is illustrated in Fig. 4.

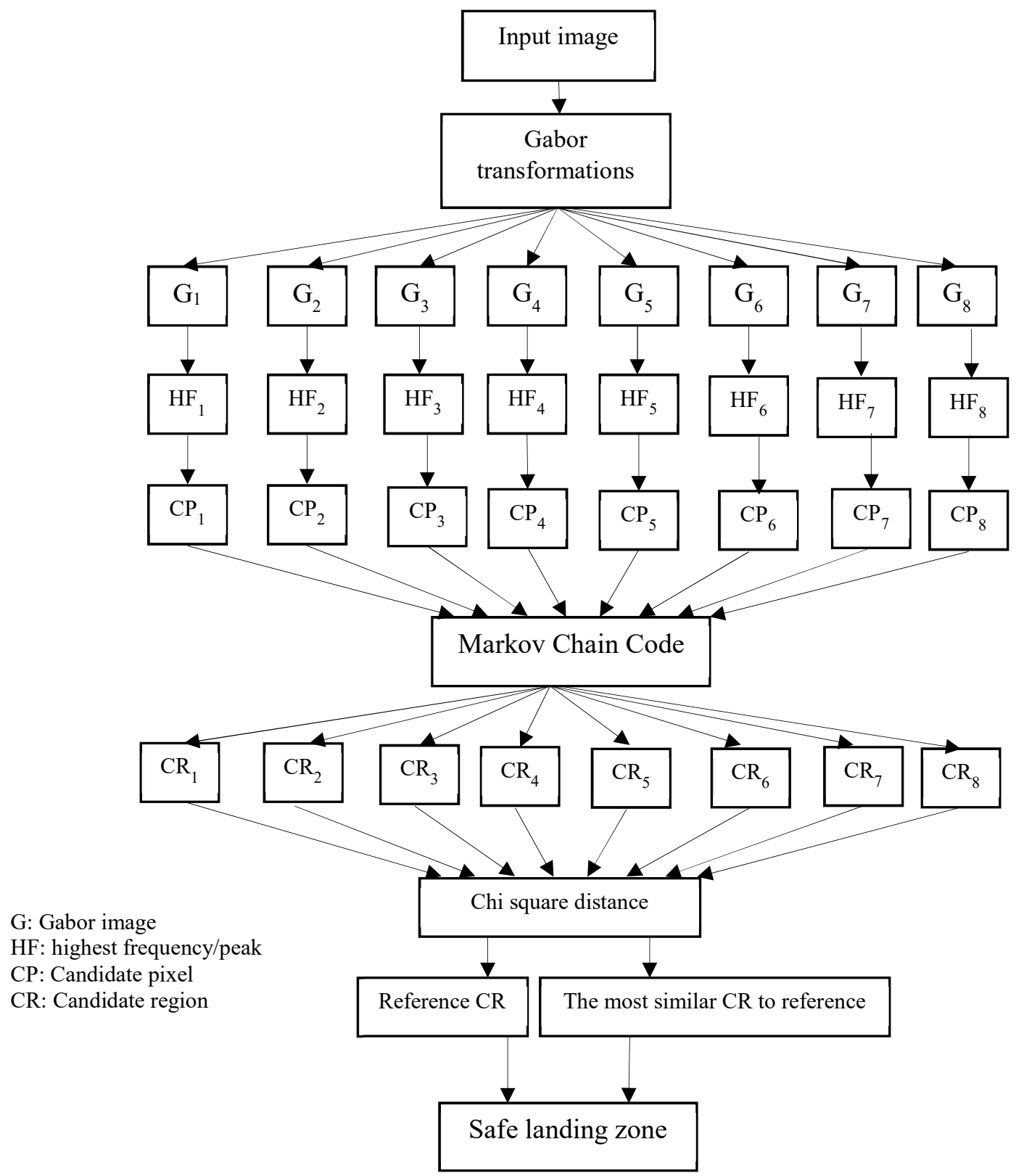

Fig. 4. Block diagram of the proposed method 


\subsection{Gabor Transform for Candidate Pixel Detection}

For each input image, the proposed system obtains eight Gabor orientations as defined in Equation (1) and the results are shown in Fig. 5, where we can see clearly that Gabor responses give almost zero for the regions which are homogenous and plain. This is understandable because of the fact that the objects in the image contain prominent edge information that spreads in 8 directions (Saxena et al., 2018). This observation leads to determine 8 equal angles from the range of 0-180 degrees, resulting in 8 Gabor responses (alpha). It ensures that the homogenous region being considered is a candidate region for finding safe landing zones, where the Gabor orientations give a zero response in all the prominent 8 directions. The number of the orientations are determined experimentally. It is evident from Fig. 5 that the Gabor orientations provide almost uniform values for homogeneous regions. The proposed system generates a histogram for the response of each pixel in images as shown in Fig. 6. To extract such regions, the proposed system selects the pixels that contribute to the highest peak in the histograms as Candidate Pixels (CPs) for the response images as shown in Fig. 7, where the white pixels represent CPs. Before performing the histogram operation, the proposed system normalizes the values to the range of zero and one as defined in Equation (2). It is observed from Fig. 7 that the above histogram operation detects other background pixels as CPs due to complex backgrounds and contrast variations. Therefore, in order to remove false CPs, we propose the Markov Chain Code (MCC), which will be discussed in the subsequent section.

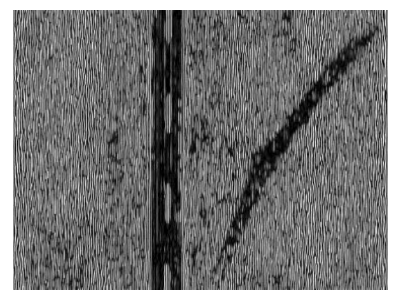

$\mathrm{G}_{1}, \alpha=0$

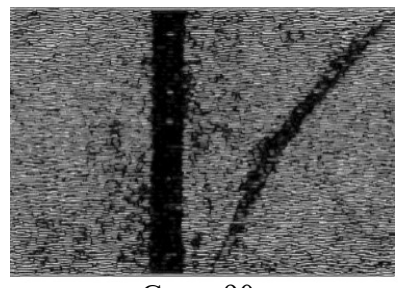

$\mathrm{G}_{5}, \alpha=90$

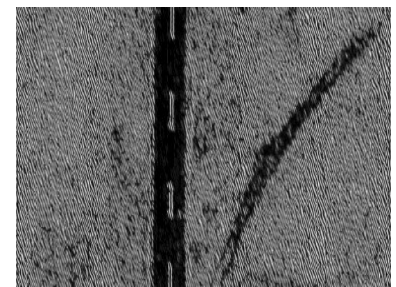

$\mathrm{G}_{2}, \alpha=22.5$

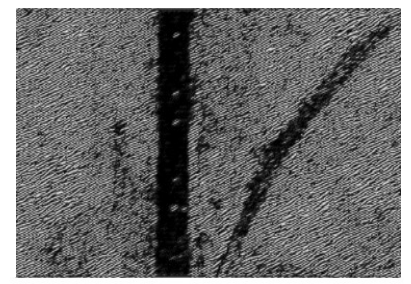

$\mathrm{G}_{6}, \alpha=112.5$

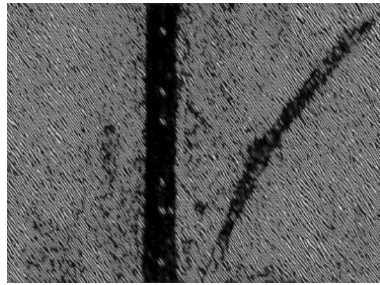

$\mathrm{G}_{3}, \alpha=45$

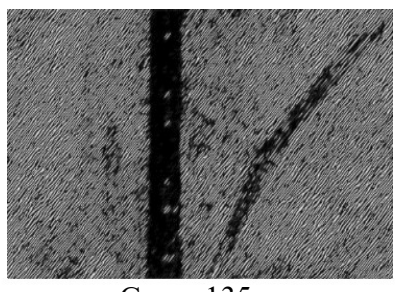

$\mathrm{G}_{7}, \alpha=135$

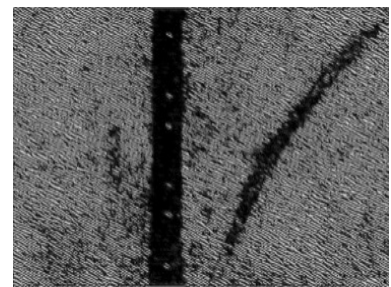

$\mathrm{G}_{4}, \alpha=67.5$

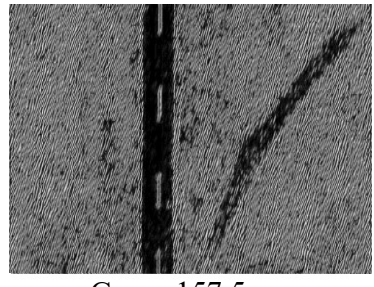

$\mathrm{G}_{8}, \alpha=157.5$

Fig .5. The response of different Gabor orientations for the first image in Fig. 3 (a). 

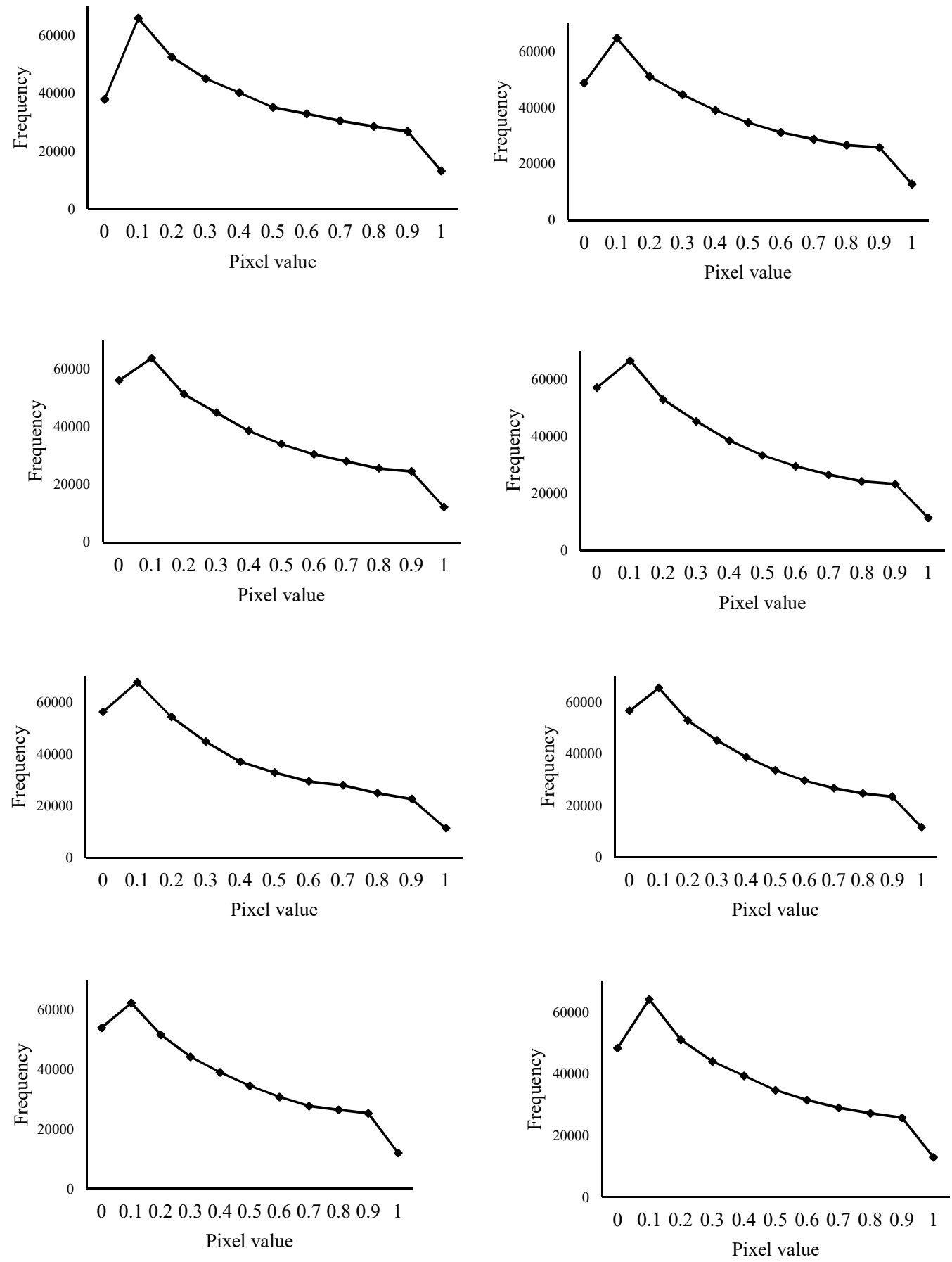

Fig. 6. Histograms for pixel values vs their frequencies to find candidate pixels from respective Gabor orientations. 


$$
\begin{gathered}
G(x, y ; f, \alpha)=\exp \left\{\frac{-1}{2}\left[\frac{x^{\prime}}{\delta_{x}^{2}}+\frac{y^{\prime}}{\delta_{y}^{2}}\right]\right\} \cos \left(2 \pi f x^{\prime}\right), \\
x^{\prime}=x \sin \alpha+y \cos \alpha, \quad y^{\prime}=x \cos \alpha-y \text { si } \alpha,
\end{gathered}
$$

where, $f$ is the frequency of the wave propagating in the direction of $\alpha$ from the x-axis, while $\delta_{x}$ and $\delta_{y}$ define the Gaussian envelope along the respective axes, respectively. More details of the steps and the implementation can be found in (Saxena et al., 2018).

$$
N\left(G_{i}(x, y)\right)=\frac{G_{i}(x, y)}{\operatorname{Max}_{G_{i}}-\operatorname{Min}_{G_{i}}}
$$

where $\operatorname{Max}_{G_{i}}$ and $\operatorname{Min}_{G_{i}}$ are the maximum and minimum values of the $i^{\text {th }}$ Gabor image, respectively. Each pixel value of $G_{i}(x, y)$ is divided by the difference between $\operatorname{Max}_{G_{i}}$ and $\operatorname{Min}_{G_{i}} . N\left(G_{i}(x, y)\right)$ is the normalized value of each pixel $G_{i}(x, y)$.
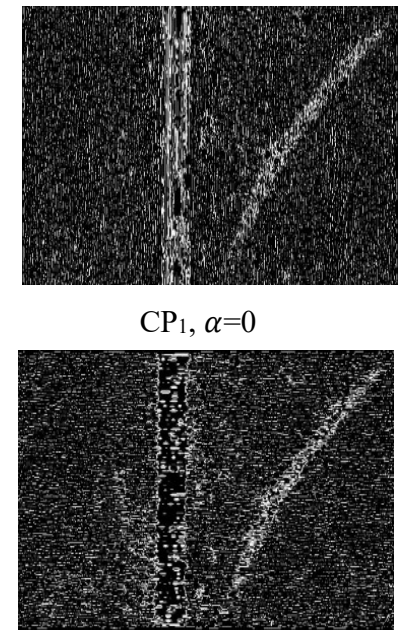

$\mathrm{CP}_{5}, \alpha=0$

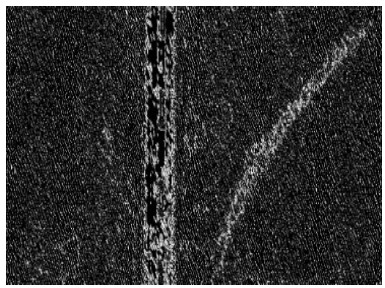

$\mathrm{CP}_{2}, \alpha=22.5$

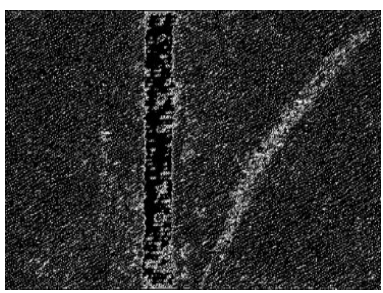

$\mathrm{CP}_{6}, \alpha=22.5$

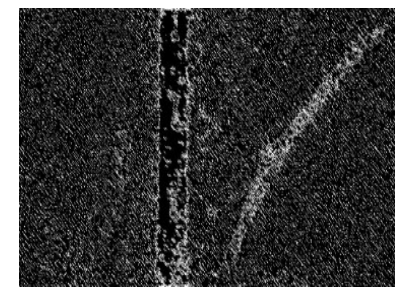

$\mathrm{CP}_{3}, \alpha=45$

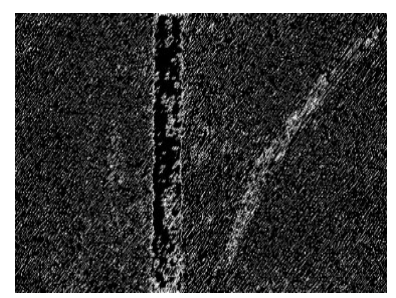

$\mathrm{CP}_{7}, \alpha=45$

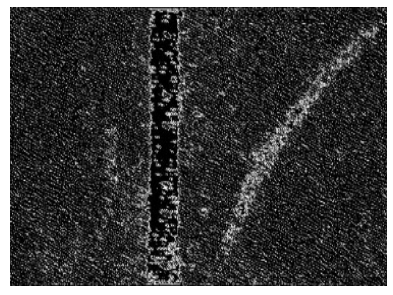

$\mathrm{CP}_{4}, \alpha=67.5$

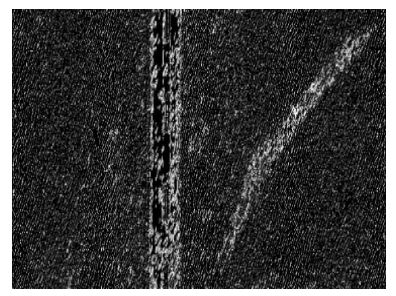

$\mathrm{CP}_{8}, \alpha=67.5$

Fig.7. Candidate pixel (white colored pixels) detection for the respective Gabor orientations in Fig. 5.

\subsection{Markov Chain Code Process for Candidate Region Detection}

As mentioned in the Proposed System Section and the previous section, a Markov Chain Code (MCC) process helps us to group the pixels that share similar values. Therefore, for each pixel and its eight neighbors, we employ MCC as defined in Equation (3)-Equation (6) for the respective response images given in the previous section. The MCC works based on the fact that the states of the pixels should be defined based on the high probabilities of neighboring pixels. The process of MCC is presented in the following. Let $S$ denote the system state at time $t$ as $S_{t}=\left\{V_{t}, N_{t}\right\}$, where $t=\{1,2\}$, which represents the assigned time for the current and improved pixel, and $V_{t} \in\{0,1\}$ represents the pixel value at time $\mathrm{t} ; N_{t} \in$ 
$\left\{0,1,2, \ldots, n^{2}\right\}$ equals the summation of the pixel and its neighboring pixels in an $n \times n$ window (since we consider eight neighbors of each pixel, $n$ is 3 ). We check each pixel $i$ of the response image for time $t=1$ (see Fig. 7) to find $S_{i 1}=\left\{V_{i 1}, N_{i 1}\right\}$, and then according to Equation (3), the next state of pixel $S_{i 2}=$ $\left\{V_{i 2}, N_{i 2}\right\}$ is determined (see Fig. 8). A probability is assigned to each pixel $i$ as defined in Equation (3)Equation (6). Since in MCC the summation of arrows exiting from a state is $1, P\left(N_{i 1}\right)+P\left(N_{i 2}\right)=1$.

$$
S_{i 2}= \begin{cases}0, N_{i 1} & \text { if } V_{i 1}=0 \text { and } P\left(N_{i 1}\right) \geq P\left(N_{i 2}\right) \\ 1, N_{i 1}+1 & \text { if } V_{i 1}=0 \text { and } P\left(N_{i 1}\right) \leq P\left(N_{i 2}\right) \\ 1, N_{i 1} & \text { if } V_{i 1}=1 \text { and } P\left(N_{i 1}\right) \geq P\left(N_{i 2}\right) \\ 0, N_{i 1}-1 & \text { if } V_{i 1}=1 \text { and } P\left(N_{i 1}\right) \leq P\left(N_{i 2}\right)\end{cases}
$$

When $V_{i 1}=0$, as the number of ' 0 's increases and therefore $N_{i 1}$ decreases, the probability that in the next state $S_{i 2}, V_{i 2}=0$ increases. Conversely, when $V_{i 1}=1$, as the number of ' 1 's and therefore $N_{i 1}$ increase, the probability that in the next state $S_{i 2}, V_{i 2}=1$ increases.

$$
\begin{array}{cc}
P\left(N_{i 1}\right)=\left\{\begin{array}{cc}
1-\beta \times N_{i 1} & \text { if } V_{i 1}=0 \\
1-\beta \times\left((n \times n)-N_{i 1}\right) & \text { if } V_{i 1}=1
\end{array}\right. \\
P\left(N_{i 2}\right)=\left\{\begin{array}{lc}
\beta \times N_{i 1} & \text { if } V_{i 1}=0 \\
\beta \times\left((n \times n)-N_{i 1}\right) & \text { if } V_{i 1}=1
\end{array}\right. \\
\beta=1 /(n \times n) &
\end{array}
$$

Since it is a two-class problem, i.e., either a pixel is to be zero or one, we consider two states for grouping the pixels as the process shows in Fig. 8(a) and Fig. 8(b), respectively, where we can see step by step the process of MCC for classifying pixels as zero or one based on probability.

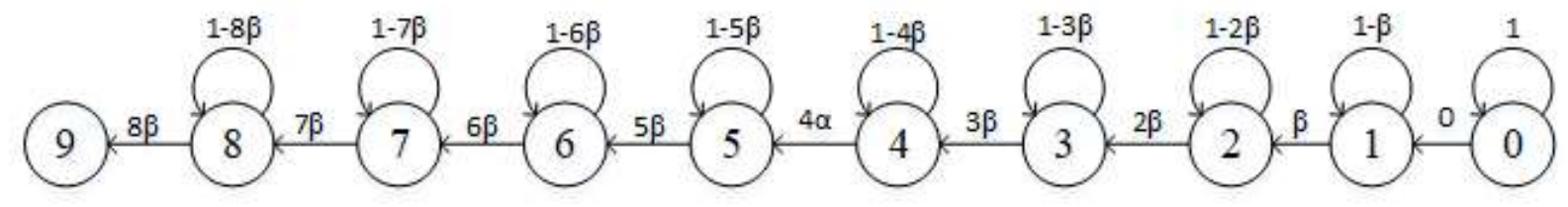

(a) Markov Chain Code for $V_{1}=0$

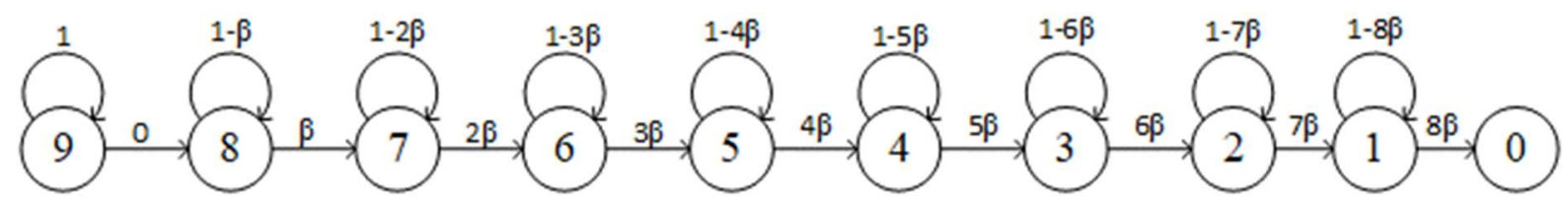

(b) Markov Chain Code for $V_{1}=1$

Fig. 8. Markov Chain Code for grouping pixels to detect candidate regions.

The effect of MCC for the respective response can be seen in Fig. 9, where it is clear that MCC helps us to cluster the pixels that share the same values based on the probability of neighbors, which results in 
Candidate Regions (CRs). It is noted from Fig. 9 that there are unwanted CRs due to the complexity of the problem. To remove such noisy components from the results of the MCC, we propose to find the largest $\mathrm{CR}$ by comparing areas of the $\mathrm{CR}$ for the respective response images, which is considered as the reference CR for each response image. Since we have different sized reference CRs for the response image, we propose an automatic criteria to calculate the threshold to classify unwanted small regions, which is independent of the CR of different response images. As a result, the area of the reference CR is compared with other CRs of the same response image based on a fixed small threshold as defined in Equation (7), where $T$ denotes the threshold and its value is 0.03 . The value of the threshold is determined empirically, which is shown in the Experimental Section. The steps to determine the threshold are as follows. Let each response image $i, i=\{1,2, . ., 8\}$, have $j$ regions $j=\{1, \ldots, \mathrm{m}\}$ called $C R_{i}^{j}$. We label them and count the number of their pixels $\operatorname{Num}\left(C R_{i}^{j}\right)$ to find the biggest region of each image called $C R_{i}^{\max }$. The next step is removing small regions to compare with the largest region. Equation (7) removes $C R_{i}^{j}$ when $L\left(C R_{i}^{j}\right)=0$ and keeps it when it equals 1 .

$$
L\left(C R_{i}^{j}\right)= \begin{cases}0 & \text { if } \operatorname{Num}\left(C R_{i}^{j}\right) \leq T \times N u m\left(C R_{i}^{\text {max }}\right) \\ 1 & \text { if } \operatorname{Num}\left(C R_{i}^{j}\right) \geq T \times N u m\left(C R_{i}^{\text {max }}\right)\end{cases}
$$

The effect of Equation (7) is shown in Fig. 10, where we can see it removes almost all the unwanted CRs. To fill the small gaps between CRs, the proposed system performs the morphological operation over the result of Equation (7) as shown in Fig. 11, where it can be seen that the gaps are filled, resulting in completely connected CRs. It is observed from Fig. 11 that one response gives a good CR and another response loses information. This is evident because of the effect of different Gabor orientations for the unexpected nature of the image. To overcome this issue and to get a complete and final CR, we estimate the degree of similarity to fuse the corresponding CRs in the response images.
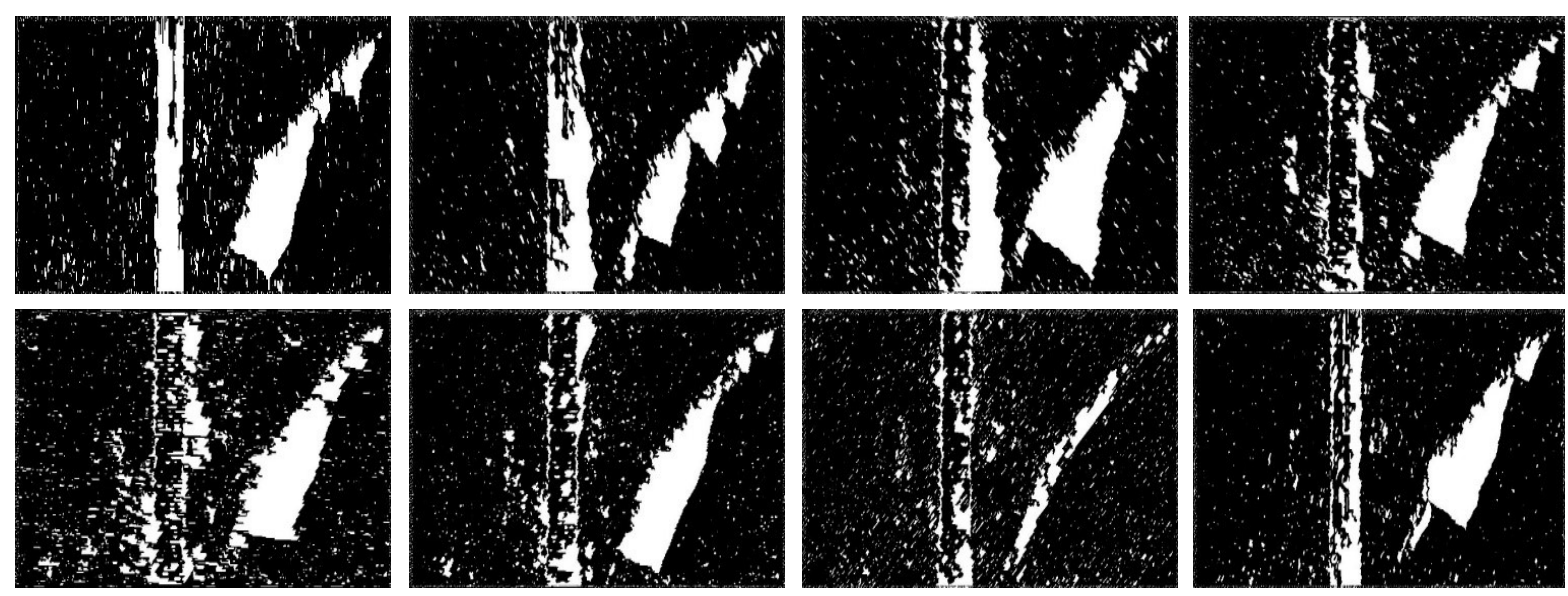

Fig. 9. Candidate regions (white colored pixels) detection using a Markov Chain Code for respective Gabor orientations. 

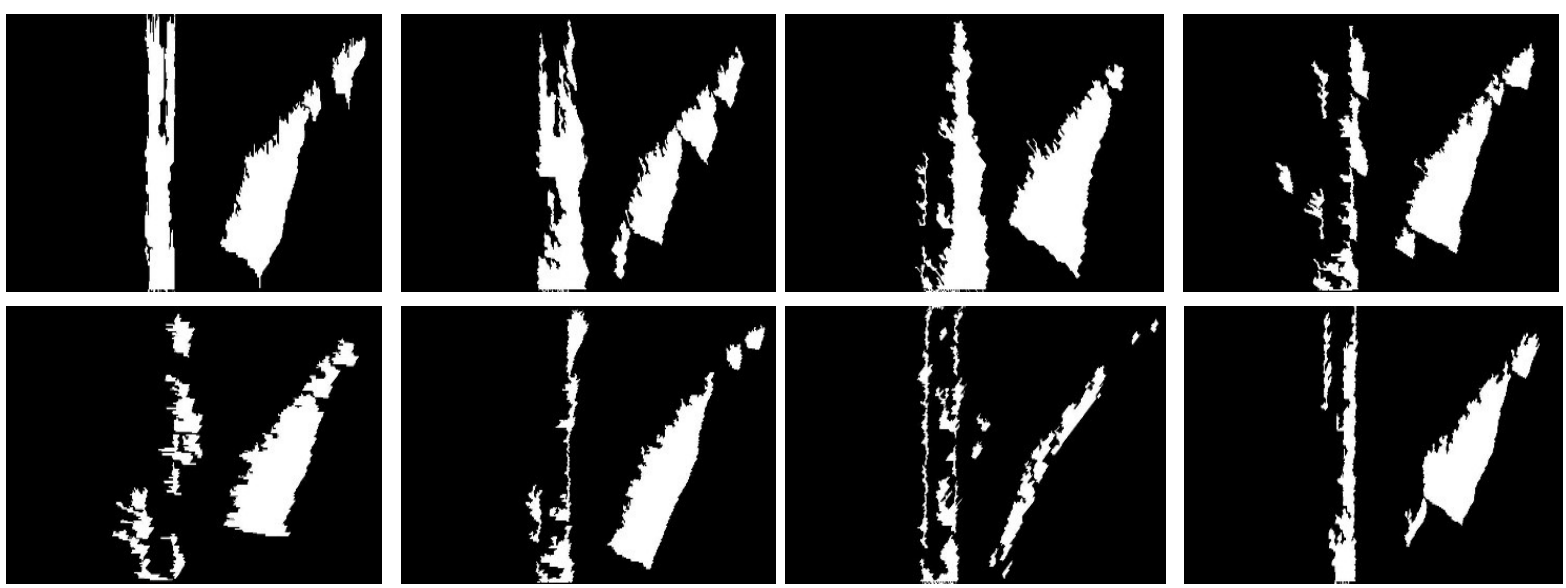

Fig.10. Candidate regions detection (white colored pixels) after removing small regions for the respective Gabor orientations.
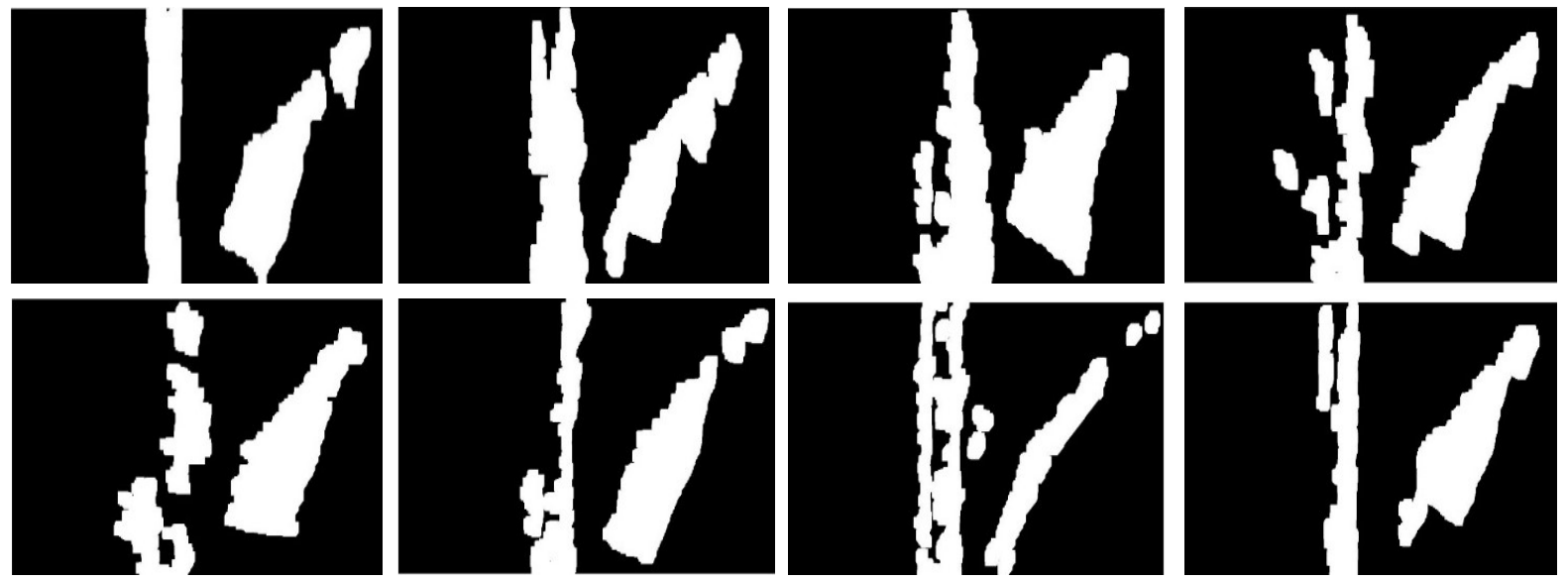

Fig. 11. Dilating candidate regions (white colored regions) to fill small gaps between the components.

\subsection{Chi Square Distance Measure for Safe Landing Zone Detection}

As discussed in the previous section, we propose to explore Chi square to estimate the degree of similarity between the reference CR and corresponding CRs of the respective response images. For each CR, including the reference CR of the respective response images as shown in Fig. 12(a), which is a reference CR for the input image in Fig. 3(a), the proposed system extracts the probability given by MCC for all the pixels of the CR. Then it generates a histogram for the probability values of the CR, which gives a distribution of pixels for each CR. Motivated by the method in (Ghosh et al., 2012), we estimate the degree of similarity between the reference $\mathrm{CR}$ and the corresponding CRs of respective responses by considering shapes of the histograms (distribution) as defined in Equation (8). Out of many CRs in the respective response images, the proposed system chooses the one which is the most similar to the reference CR as shown in Fig. 12(b). The proposed system performs a union operation for fusing the reference CR with the most similar CR as shown in Fig. 12(c), where we can see the complete CR region. In the same way, the 
process continues for other CRs corresponding with the respective response images. The final result for the input image is shown in Fig. 12(d), where we can see two CRs which can be used for safe landing of UAVs for the input image in Fig. 3(a). The formal steps for the above process are presented as follows.

Let the $j^{\text {th }}$ candidate region of the $i^{\text {th }}$ response image be considered as a reference region, called $C R_{i=r}^{j}$, for example, $C R_{3}^{1}$, as shown in Fig. 12(a). For all $C R_{i}^{1} i=\{1,2, . ., 8\}$, we perform a histogram operation, $H_{C R_{i}^{j}}$ with $\partial$ bins for the probability values given by MCC as discussed in Section 3.2. In order to study the shape of the histogram distribution, the proposed system finds the absolute difference between frequency values of the successive bins in the histogram, which outputs a new histogram $H^{\prime}$ as defined in Equation (8), where $\partial=\{1,2, . ., 10\}$. In this work, we consider a histogram with 10 bins as it gives promising results.

$$
\left(H_{C R_{i}^{j}}^{\prime}\right)_{\partial}=\left|\left(H_{C R_{i}^{j}}\right)_{\partial}-\left(H_{C R_{i}^{j}}\right)_{\partial+1}\right|
$$

Equation (9) is the $\mathrm{X}^{2}$-test that is a statistical method of determining the similarity between two distributions of regions. This Equation calculates the difference between the histograms of the reference candidate regions $H_{C R_{r}^{j}}^{j}$ with other candidate regions in the respective Gabor response images $H_{C R_{i}^{j}}^{\prime}$. According to this, the calculation $C R_{1}^{1}$ is determined as the most similar region to the reference as shown in Fig. 12(b).

$$
X^{2}\left(H_{C R_{r}^{\prime}}^{\prime}, H_{C R_{i}^{\prime}{ }_{i \neq r}}\right)=\sum \frac{\left(\left(H^{\prime}{ }_{C R_{r}^{j}}\right)_{\partial}-\left(H^{\prime}{ }_{C R_{i}^{j}}\right)_{\partial}\right)^{2}}{\left(\left(H^{\prime}{ }_{C R_{r}^{j}}\right)_{\partial}+\left(H^{\prime}{ }_{C R_{i}^{j}}\right)_{\partial}\right)}
$$

The same process continues for the second candidate region of reference $C R_{r}^{2}$ and the process terminates when it has visited all the CRs in the image.

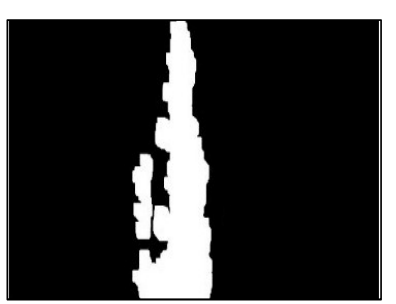

(a) Reference CR

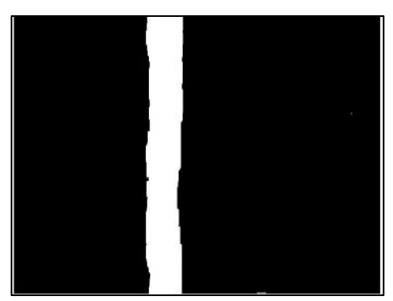

(b) The most similar CR among 8 CRs

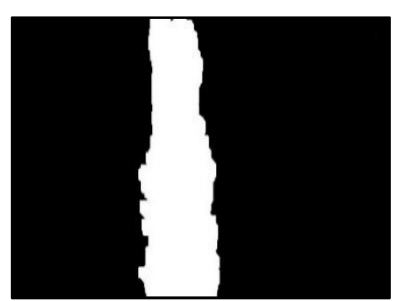

(c) Fusing (a) and (b)

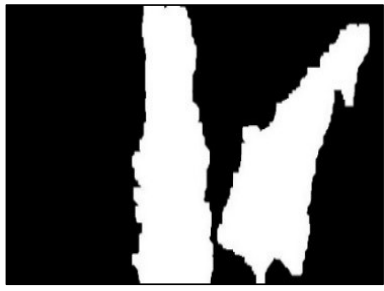

(d) Safe land region

Fig 12. Fusion process for finding a safe land region (white colored region) from eight Gabor oriented images.

The proposed system consists of two parts, namely, for the given input image, the method detects candidate regions and then chooses relevant regions for the safe landing of a UAV according to emergency scenarios. In this work, we consider three common and sensitive dangerous situations, which may cause emergency 
landings, namely, issues with communications, GPS and software-hardware/energy errors (Patterson et al., 2014). The details for each cause are as follows.

Case-1: A communication link enables the UAV to be controlled via sending and receiving essential commands from a human-in-the-loop scenario. The loss of this wireless link would make the UAV out of control and would result in the occurrence of undesirable critical events. In this situation, in addition to general safety needs, the most important criterion is to choose a safe landing zone, which is near to a transmitter station (TS) to increase the chance of reconnection.

Case-2: A vital part of a UAV sensing system is the GPS. It provides the UAV with essential information. Although, studies mostly assume that it is always available and accurate, there are still limitations in real situations, such as the unavailability of satellite services, errors and uncertainties related to atmospheric layers, poor space weather conditions, physical obstacles and blocks in outdoor or indoor environments, as well as very high latitude. In this case, the most important landing issue is being far from tall obstacles $(O)$ such as mountains, buildings and threats that may cause collisions.

Case-3: Sometimes, one can encounter unexpected software and hardware errors, for example, actuator failure or software module crashes that force the UAV to land as soon as possible to the nearest available region. In addition, UAVs may face very low power availability because of uncertainties in real environments, which would prevent it from returning to its base station and forcing a landing at the nearest opportunity. In this situation, the main concern would be for navigation towards the nearest detected (safe) zone.

\section{Experimental Results}

This section is divided into four sub-sections. The descriptions of the dataset and evaluation criteria for measuring the performance of the proposed and existing methods are given in Section 4.1. The empirical analysis for determining the values of key parameters used in the proposed method is presented in Section 4.2. Section 4.3 presents evaluation of the safe landing zone detection step, and Section 4.4 provides validation for the method of safe landing zone detection.

\subsection{Dataset and Evaluation}

There is no standard dataset which can be used for evaluating the proposed system. Therefore, we create our own dataset which includes images captured by a custom-build quadcopter and also Google images. In total, we collected 50 images for experimentation and evaluation in this work. The dataset consists of images of different situations, such as roads, trees, lawn, grass, forest and buildings. In addition, the dataset includes images captured by drones, which may fly in the range of 30 to 2000 meters with reference to the 
ground station. We believe the dataset created comprises the possible situations for emergency landing of a UAV, as mentioned in the previous section.

In order to show the superiority compared to existing systems, we implemented the state-of-the-art method, namely, (Patterson et al., 2014), which explores edge-based features for potential zone detection. It also proposes a fuzzy-based method for classifying potential zones into categories of risky, safe and unsafe. In addition, we considered Aziz et al.'s method (2016), which explores edge information and histogram operations to classify the given images into 'good' or 'bad' classes. The reason to choose these two methods for comparative studies with the proposed system is that the objectives of those existing systems are the same as the proposed system, i.e. the segmentation of regions and then presenting the usefulness of the segmented regions for safe landing of UAVs. Note: since Patterson et al.'s method (2014) outputs three classes, namely, risky, unsafe and safe, for each given input image, we considered the images that were classified as safe for calculating the measures in this work. Similarly, since Aziz et al.'s method (2016) considers the whole image as the input for the classification of 'good' or 'bad' for UAV landing, we passed the regions segmented by the proposed system as the inputs to the existing system to calculate the relative successful and unsuccessful rates. In other words, for each image, the proposed system obtains Candidate Regions (CRs) and Non-Candidate Regions (Non-CRs). For these two regions, if an existing system classifies candidate regions as 'good' and non-candidate regions as 'bad', it will be considered as a successful segmentation, otherwise it is an unsuccessful segmentation. To evaluate the proposed and existing systems for the segmentation of safe zones for UAV landing, we used well-known measures, namely, Recall, Precision and F-measure as defined in Equation (10)-Equation (12).

$$
\begin{gathered}
\text { Recall }=\frac{t p}{t p+f n} \\
\text { Precision }=\frac{t p}{t p+f} \\
F-\text { Measure }=2 \times \frac{\text { Precision } \times \text { Recall }}{\text { Precision }+ \text { Recall }}
\end{gathered}
$$

As noted in the Proposed System Section, one can expect several safe lands for one image, which can be used for landing a UAV safely. As a result, for a large number of input images, the proposed system outputs a large number of safe landing regions. This leads us to select the possible relevant safe landing sites according to the above three cases. Hence, in this work, we estimate the cost by assigning weights for different factors, such as distance from UAV and distance from terrain threat situations based on predefined experiments as defined in Equation (13) and Equation (14).

The overall cost function assigned for each safe region $C R_{j} \mathrm{j}=\{1,2, . ., \mathrm{K}\}$ is $\mathrm{F}\left(C R_{j}\right)$. The objective is to find the $j^{\text {th }}$ region that gives the minimum cost according to Equation (13), where $a, b, c$ and $d$ are constant and 
the sum of those values should satisfy $a+b+c+d=1$. Let $D_{R_{j}}^{u}, D_{R_{j}}^{T S}$ and $D_{R_{j}}^{O}$ represent the Euclidean distance between the UAV and the $j^{\text {th }}$ safe region, a transmitter station and tall obstacles, respectively. $D_{\max }$ is the farthest distance in the image to the UAV in order to normalize values. Finally, $T h_{R_{j}}$ is a parameter which represents the changes according to the influence of terrain threat on the $j^{\text {th }}$ region. It is a summation of $n$ threat sources calculated as defined in Equation (14) (Huang et al., 2016), where $L_{n}$ represents the threat level of the $n^{\text {th }}$ threat, $k$ is a coefficient, and $D_{R_{j}}^{n}$ is the Euclidean distance between that threat and the region. It is divided by a maximum threat influence where threat level $L_{n_{\max }}$ is the maximum threat level and the distance of the region and a threat is at a minimum called $D_{\min }$.

$$
\begin{gathered}
F\left(R_{j}\right)=a \times \frac{D_{R_{j}}^{u}}{D_{\max }^{u}}+b \times \frac{D_{R_{j}}^{T S}}{D_{\max }^{T S}}+c \times \frac{1}{D_{R_{j}}^{O} / D_{\max }^{O}}+d \times \frac{T h_{R_{j}}}{\sum_{i=1}^{n} L_{n_{\max }} e^{-k D_{\min }^{n}}} \\
T h_{R_{j}}=\sum_{i=1}^{n} L_{n} e^{-k D_{R_{j}}^{n}}
\end{gathered}
$$

The above equations indicate that when the system gets different emergencies, higher values are assigned to the corresponding constant. Finally, the $j^{\text {th }}$ region which minimizes the cost is considered as a safe landing zone out of many candidate regions given by the segmentation step. Since there is no ground truth for evaluating the proposed system, we manually count the outputs of the proposed system to calculate relative successful and unsuccessful rates as defined in Equation (15), where $S$ is the number of successful safe regions chosen according to various situations, and $N E$ is the number of all possible emergency cases.

$$
\text { Relative Success Rate }=\frac{S}{N E}
$$

\subsection{Empirical Analysis for Determining the Values of the Key parameters}

The proposed system consists of key parameters, namely, different orientations for Gabor response in Section 3.1, the number of neighbors for the Markov Chain Code process to group the pixels which share the same values in Section 3.2, and the value of the threshold for removing noise candidate regions automatically by denoting areas of reference candidate regions in Section 3.2. To determine the values for the above three parameters, we use predefined samples chosen randomly from our database for experimentation by calculating the F-measure (F) by varying parameter values as shown in Fig. 13-Fig. 15, respectively. It is noted from Fig. 13 that as the number of the angle changes on the $\mathrm{X}$ axis, the F-measure on the $\mathrm{Y}$ axis for Candidate Region segmentation changes. However, for the number of angle (8), the proposed segmentation score is the highest and hence 8 is considered as the best number in this work. It is valid because for a smaller number of angles, there is a chance of losing salient information and for a larger number of angles, there is the potential of introducing redundancy. 


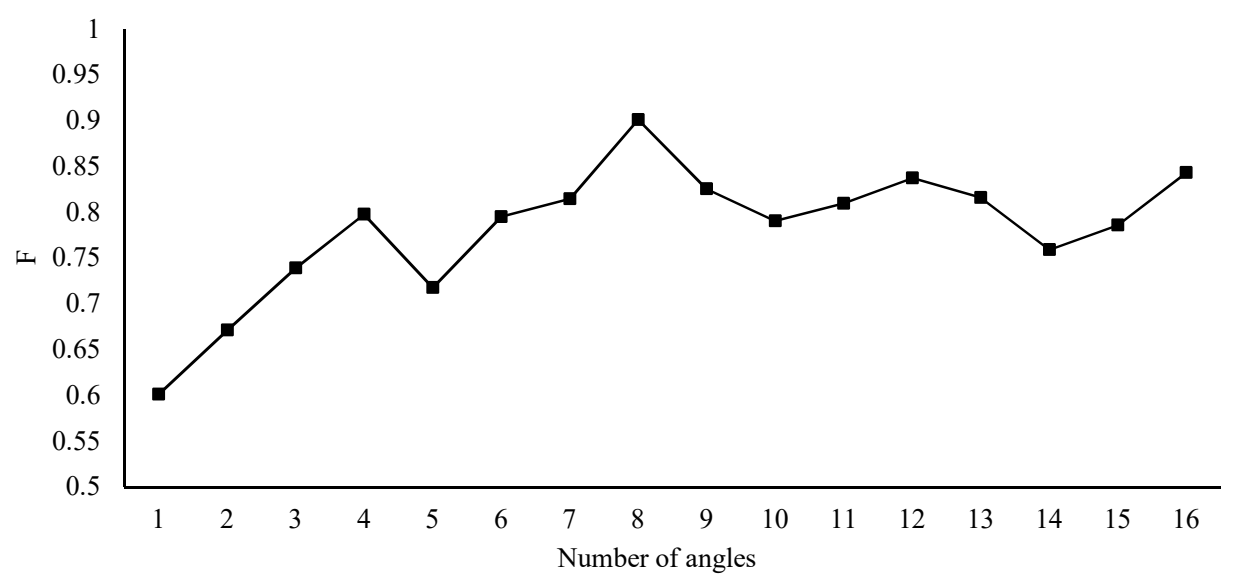

Fig. 13. F-measure for different Gabor orientations to determine the best Gabor responses.

Similarly, we conduct experiments on the same predefined data for determining the best number of neighbors to group the pixels using the Markov Chain Code as shown in Fig. 14, where we can see that as the window size increases, the performance of segmentation decreases. However, for the neighbors of 8 , the proposed segmentation scores the highest F-score and thus 8 neighbors $(n=3)$ is considered to be the best configuration. It is justifiable because when we increase the size of the neighbors, there is a chance of losing pertinent information. In addition, it will be a computationally expensive process.

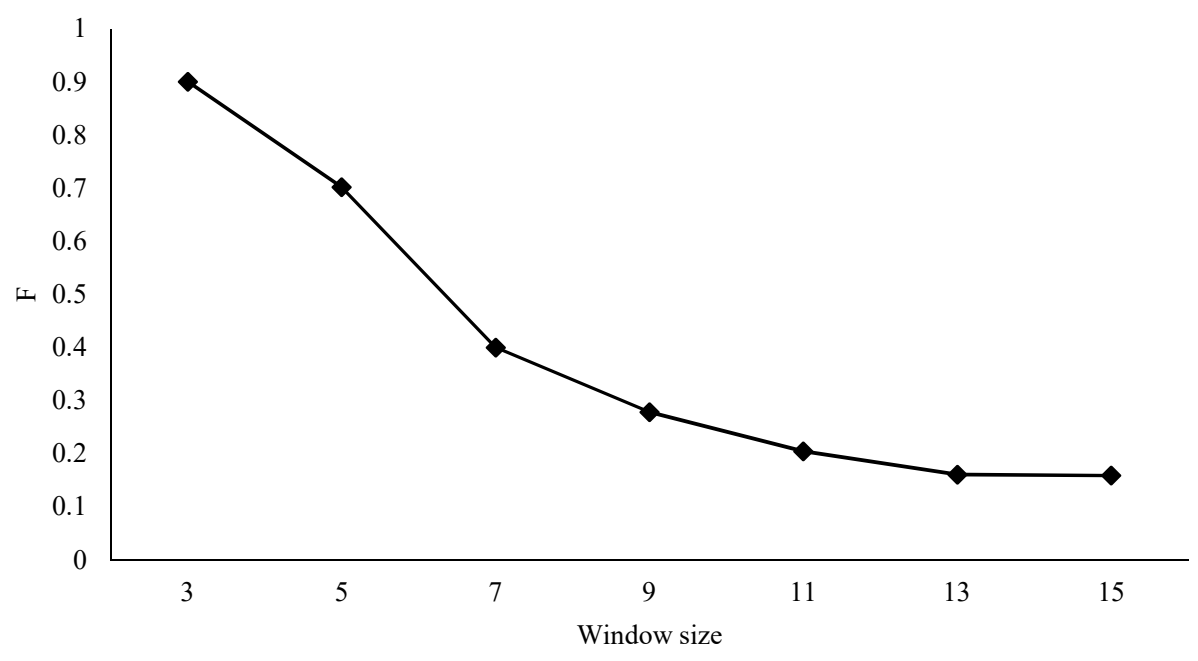

Fig. 14. F-measure for different window sizes to determine the best candidate regions 
In the same way, the value of the threshold is determined for removing unwanted candidate regions, which is independent of the candidate regions in the respective response images as noted in the experiments shown in Fig. 15, where it can be seen that as the threshold value increases, the performance of segmentation decreases. At the 0.03 value, the performance reaches the highest level and thus 0.03 is considered as the optimum configuration for achieving better results. This is because when we increase the threshold value, there is a chance of losing significant candidate regions in different response images. If it is less than 0.03 , there is the possibility of increasing false candidate regions.

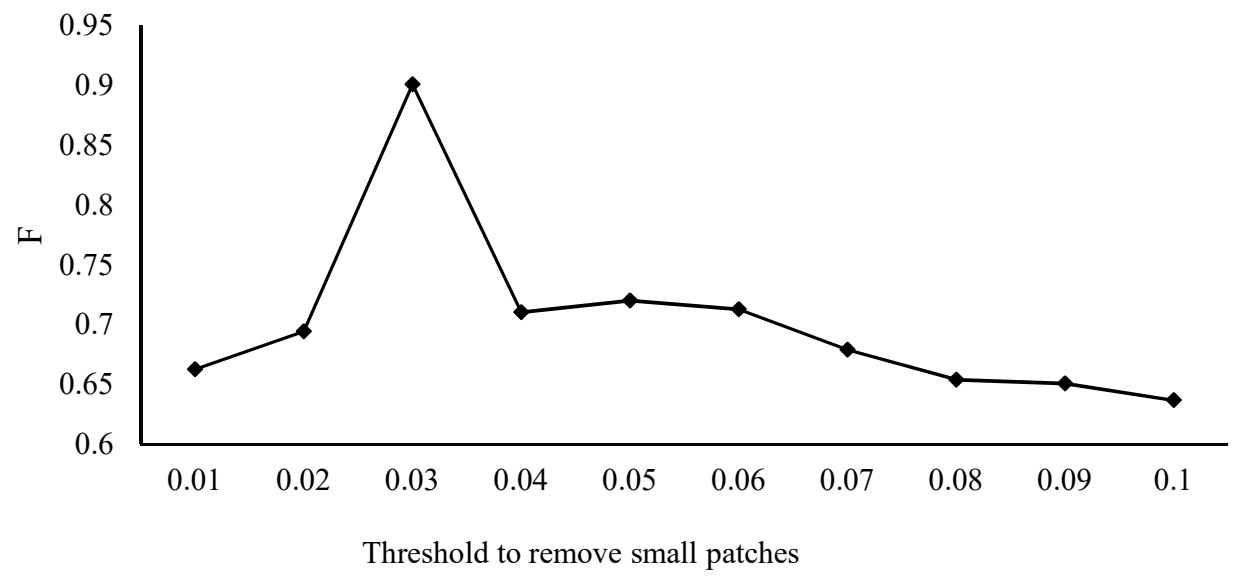

Fig. 15. F-measure for different threshold values to determine the optimum value for removing small patches

\subsection{Experiments for Evaluating Safe Landing Zone Detection}

Qualitative results of the proposed and existing systems for safe landing zone detection of UAVs are shown in Fig. 16, where we can see input images of different situatons in Fig. 16(a), and the results of the proposed system, Patterson et al.'s system (2014) and Aziz et al.'s system (2016) in Fig. 16(b), Fig. 16(c) and Fig. 16(d), respectively. It is observed from the results in Fig. 16 that the proposed system detects safe landing regions marked with a white color successfully for all the input images, while the existing systems fail to detect these accurately. For the last image, the proposed system does not detect the road as a safe landing zone due to vehicles which appear on it. Therefore, we can conclude that the proposoed system detects safe landing regions well for complex situations. The reason that the existing systems report poor results is that they are not robust and consider limited situations. It is evident that the existing systems define specific features to segment regions, such as edge-based and geometrical-based features, while the proposoed sytem considers features which represent homogenous and flat surfaces by exploring Gabor orientations. The same thing is true for the quantitative results reported in Table 1, where the proposoed system achieves the best at Recall, Precision and F-measure compared to both existing systems. This is due to generalised 
features of the proposed system without many constraints and assumptions. The reason for the poor results of the existing systems is the same as mentioned above. When we compare the results among existing systems, Aziz et al.'s method (2016) gives better results in terms of Recall and F-measure. This is because Aziz et al.'s method (2016) involves histogram operations to find homogenous regions, which is robust compared to the Canny edges used by Patterson et al. (2014), as Canny edges are sensitive to complex backgrounds.
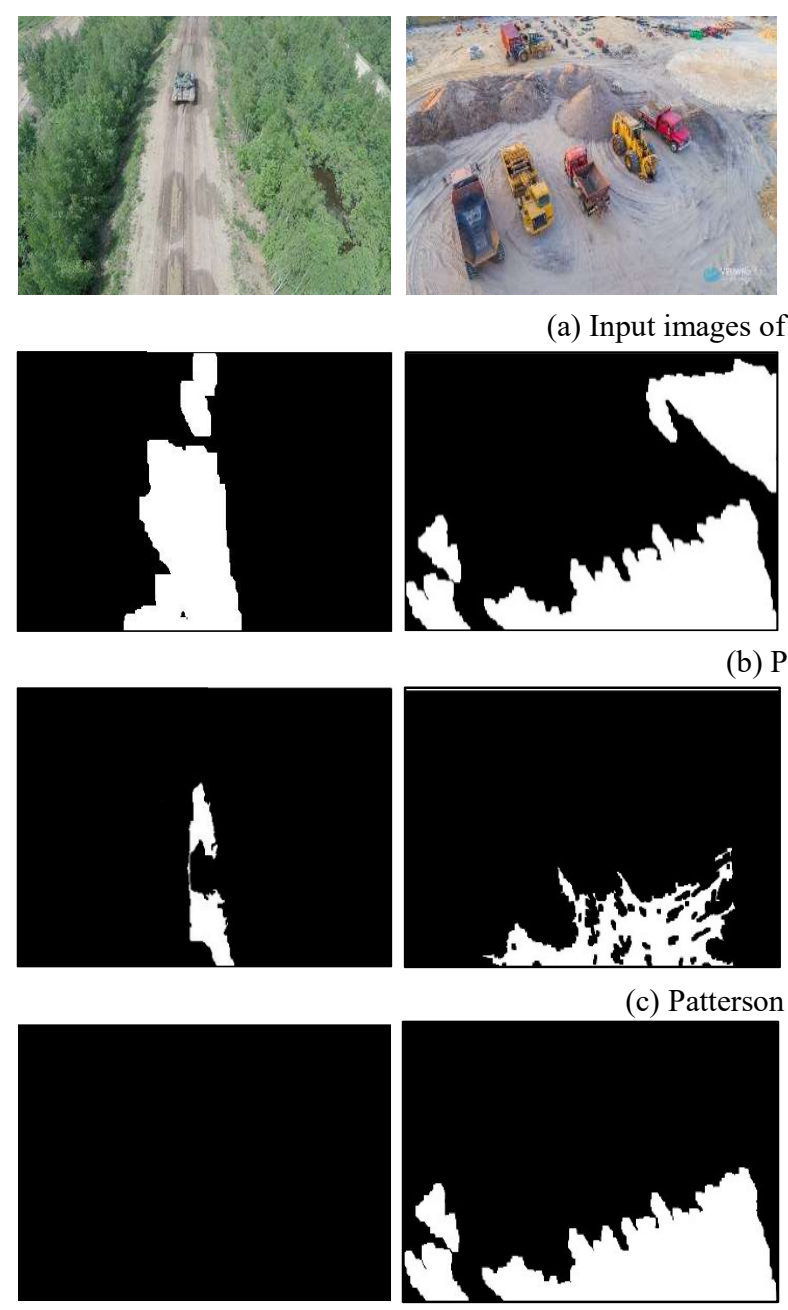

(d) Aziz et al.'s system (2016)

(a) Input images of different situations.

(b) Proposed system

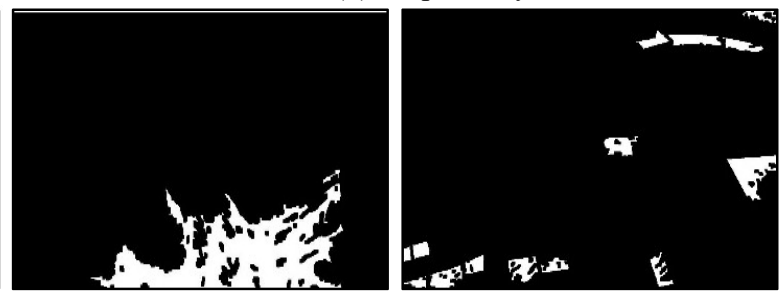

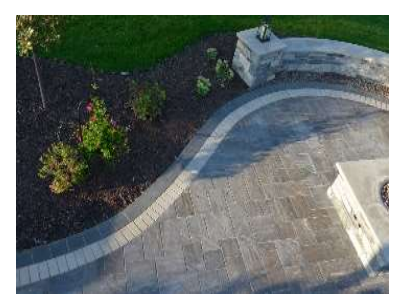
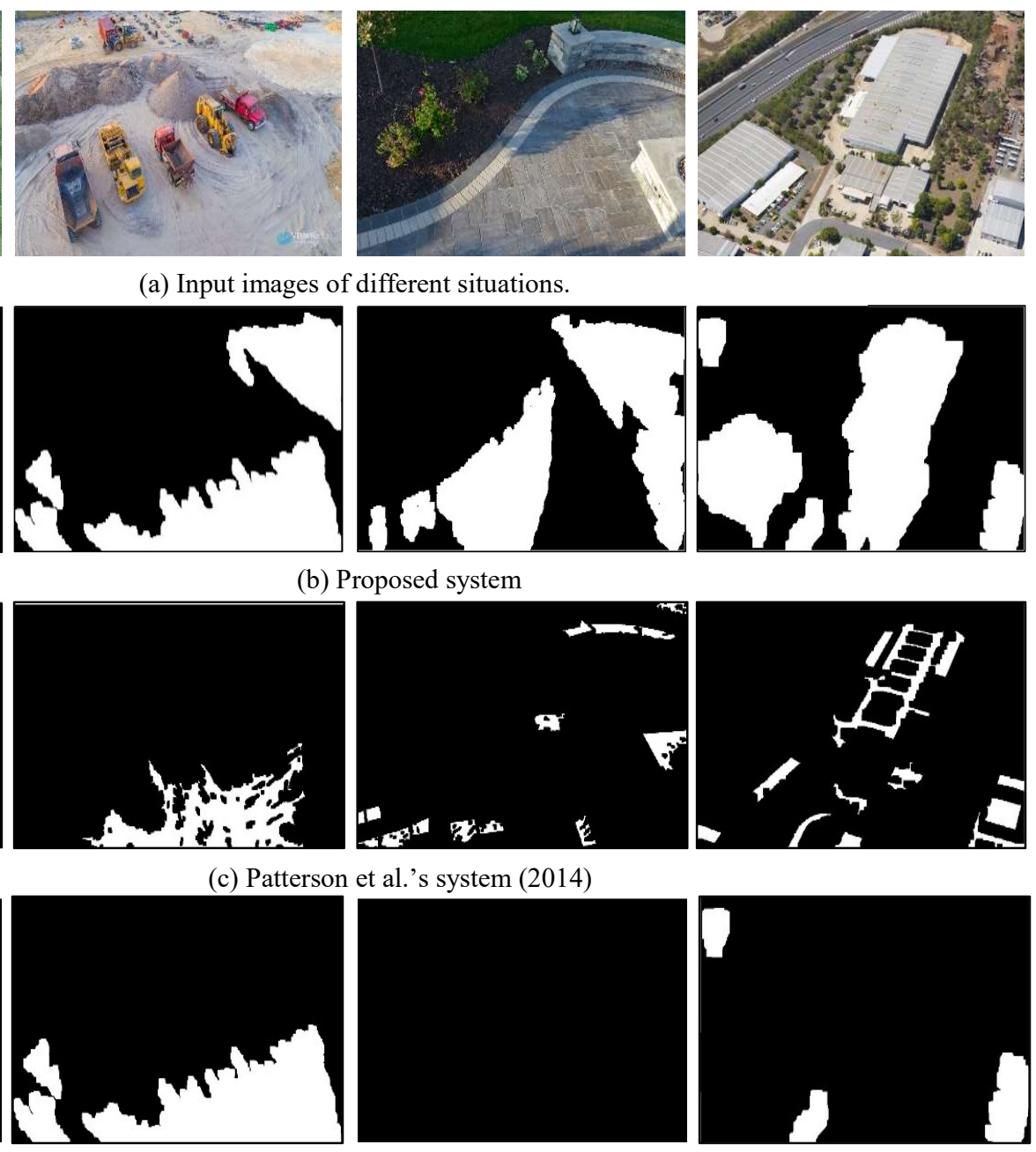

Fig. 16. Qualitative results for safe land detection (white colored regions) of the proposed and existing systems.

Table 1. Performance of the proposed and existing systems for safe landing zone detection

\begin{tabular}{|c|c|c|c|}
\hline & Patterson et al. (2014) & Aziz et al. (2016) & Proposed System \\
\hline Recall & 0.46 & 0.61 & $\mathbf{0 . 8 7}$ \\
\hline Precision & 0.93 & 0.90 & $\mathbf{0 . 9 3}$ \\
\hline F-Measure & 0.62 & 0.72 & $\mathbf{0 . 9 0}$ \\
\hline
\end{tabular}




\subsection{Experiments for Validating Safe Landing Zone Detection}

The proposed segmentation step gives several regions to enable UAVs to land safely. According to the emergency situations we mentioned earlier, the proposed system has to choose the correct region for landing from many safe landing spots. The experimental setup includes 500 random scenarios, where we create artificial threats and segments (CRs) such that the created scenario reflects a real networked environment (Huang et al., 2016). The proposed system calculates relative success rates as defined in Equation (15) based on cost estimation defined by Equation (13). It is true that the number of segments and the number of threats are key parameters, which affect success rates of the proposed system. Therefore, we set a threat level of 0.1 initially for conducting experiments to determine the number of segments as shown in Fig. 17, whereas when the number of segments increases, the success rate on the $\mathrm{Y}$ axis also increases up to 20 segments, after which the relative success rate reaches a steady state. This shows that after 20 segments, there is not much improvement in the relative success rate. This is justified, because usually in the case of a real environment, the number of segments represents safe lands given the proposed step increase, thus the system gets more chances for selecting the best one, and hence the relative success rate either increases or it would tend toward a steady state. Therefore, we consider the number of segments as 20 for evaluating the proposed system.

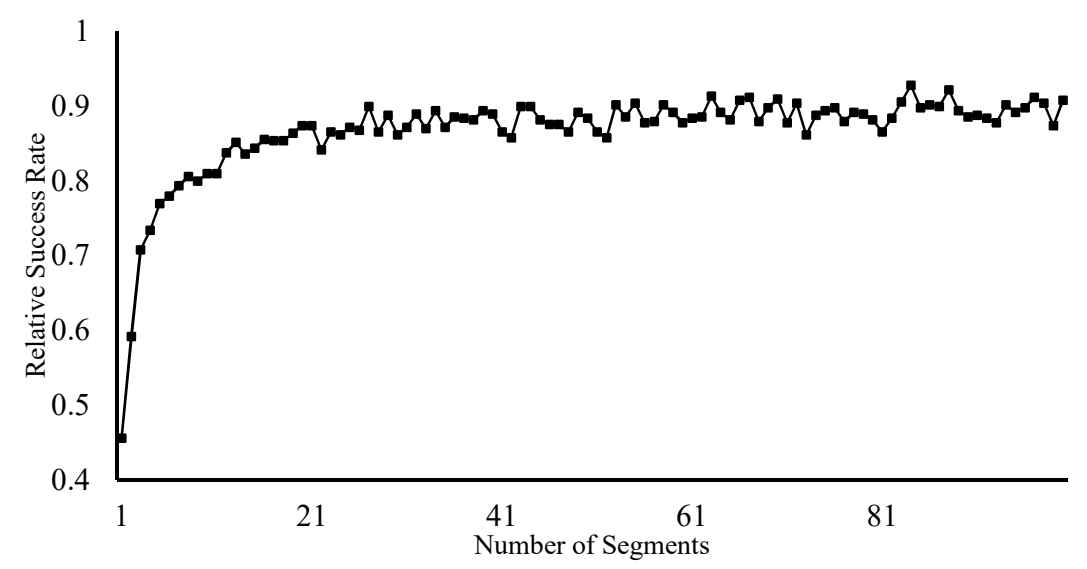

Fig. 17. Choosing an optimal number of safe lands for assessing safe landing performance

Similarly, to determine the relative threat level, we fix the number of segment candidate regions as 20 and then conduct experiments for different threat levels as shown in Fig. 18, where we can see that as the relative threat level increases, the performance of the system decreases. Note that threat indicates obstacles which prevent the UAV from landing at the zone in spite of it being detected by the proposed system. For instance, when power cables, mountains, towers, or tall buildings are near the detected safe zone, the UAV cannot choose it for landing. This is because it may lead to a collision. Therefore, when the number of such relative threats increases, the proposed system misses safe zones for landing and hence there is a poor 
relative success rate when there is a higher number of threats. Therefore, in this work, we consider 0.1 as the threat level and 20 as the number of segments (safe lands) for calculating the final relative success rates of the proposed and existing systems.

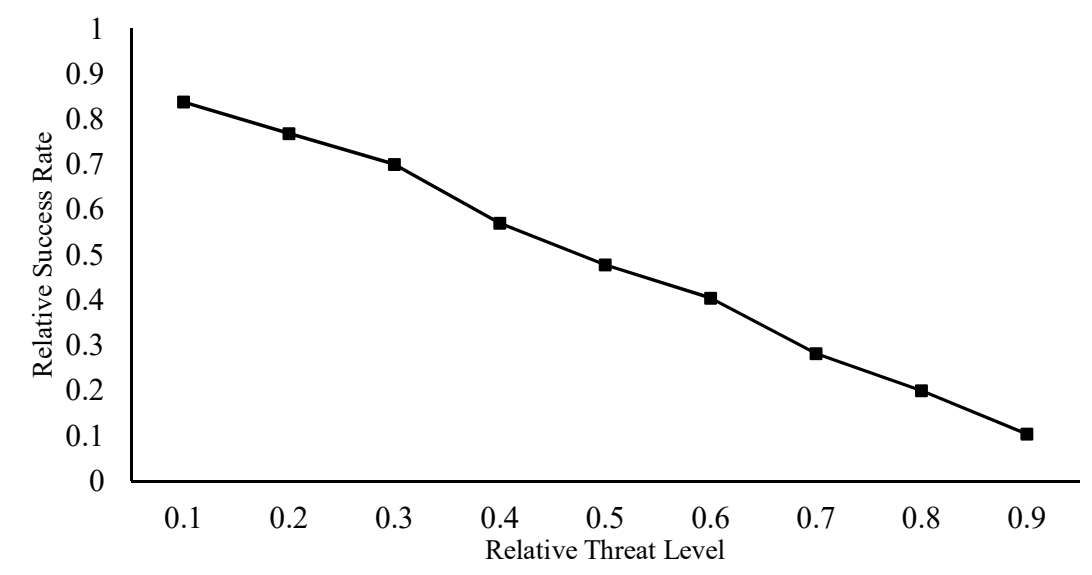

Fig. 18. Safe landing performance as relative threat level increases after fixing the number of safe

The simulation results of the proposed and existing systems on 500 random scenarios by fixing the number of segments (safe regions) as 20 and threat level as 0.1 are presented in Fig. 19. The proposed system has the highest relative success rate compared to the existing systems. In this experiment, we estimate the cost as defined in Equation (13) for calculating relative success rates. Since the proposed system segments regions as successful candidates for landing a UAV safely, there is no need for classification as in the existing systems (Patterson et al. (2014); Aziz et al. (2016)). Therefore, we estimate the cost according to the emergency type, namely, communication errors (case-1), GPS errors (case-2) and software-hardware error/energy constraints (case-3) for calculating relative success rates separately as shown in Fig. 19 for the proposed and existing systems. The key reason that the existing systems have poor relative success rates as compared to the proposed system is that the existing systems are not capable of classifying complex situations as they do not propose generalized features for segmentation. In addition, the success of classification depends heavily on the number of samples and classifier training. On the other hand, the proposed system considers generalized features for candidate region detection without classification, which contributes to its success. Note: when we compared the segmentation and classification results of the existing systems, for segmentation, Aziz et al.'s method (2016) scores best compared to Patterson et al.'s method (2014), but it is vice versa for the classification results. Aziz et al.'s method (2016) does not have robust classification results as compared to Patterson et al.'s method (2014), which considers fuzzy-based features for classification. The fuzzy-based features are effective when there is ambiguity in classification due to the large number of CRs. 


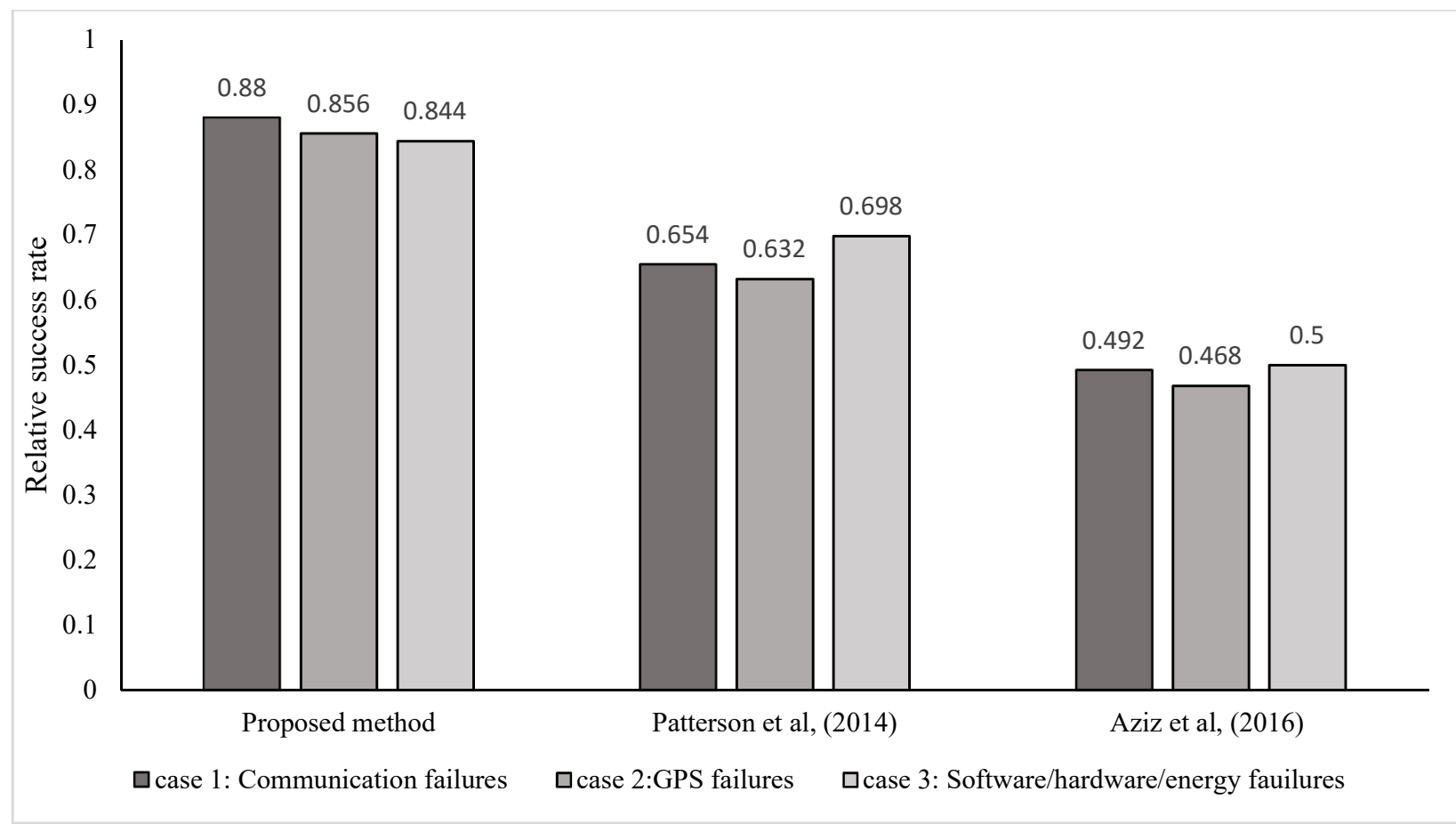

Fig. 19. Relative Success rate of the proposed and existing methods for safe landing.

Though the proposed system works well for complex situations, sometimes, it detects regions as candidate ones in images containing water. This is because the surface of the water appears as homogenous, and homogeneity is the key feature for the proposed system. In addition, sometimes, if an image contains vehicles and is captured from a long distance (height), the proposed system does not respond correctly. Therefore, there is scope for improvement in the future.
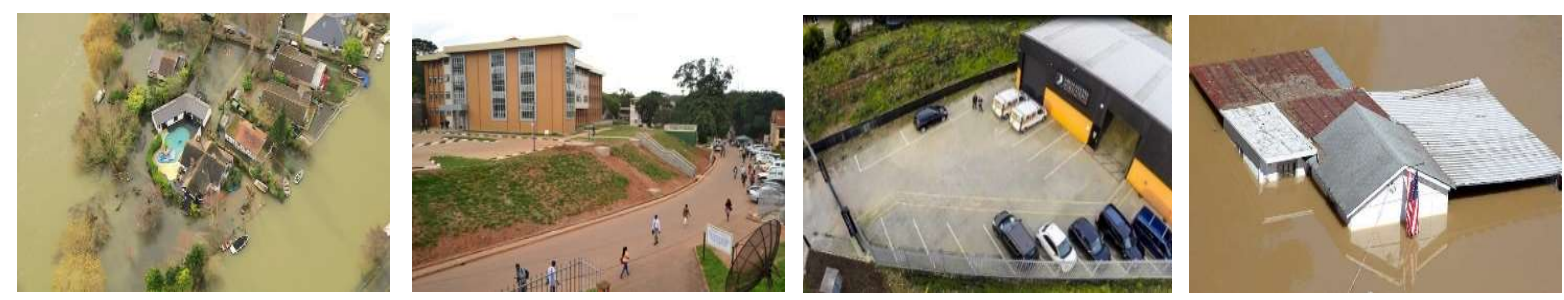

(a) Input images of different situations.
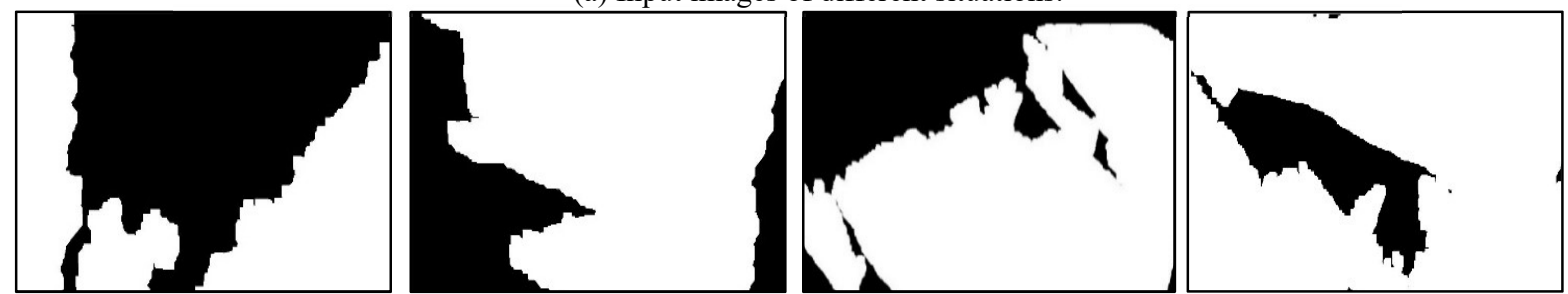

(b) Safe landing detection (white colored regions) of the proposed system

Fig. 20. Erroneous results of the proposed method for safe landing zone detection 


\section{Conclusions and Future Work}

In this work, we have proposed a new system for candidate region detection, which can be used for safe UAV landing when there is an emergency. The proposed system explores different Gabor orientations based on the fact that the degree of Gabor orientation responses is high for variations and low for smooth/flat regions in images. The same observation has been explored for finding homogeneous regions in this work, which are called flat regions for landing UAVs safely. The proposed system performs histogram operations for Gabor response images to classify pixels which contribute to homogenous regions. This results in candidate pixel detection. The proposed system introduces a Markov Chain Code for grouping neighboring pixels that share the same values based on probabilities, which result in candidate regions. Due to complex backgrounds and contrast variations, there is potential for detecting false candidate regions. To overcome this issue, the proposed system finds candidate regions using areas, which are the largest among other candidate regions corresponding to respective Gabor response images. The system compares the areas of other candidate regions to eliminate noise and small regions, which results in reference candidate regions. Furthermore, the proposed system uses Chi-square to estimate the degree of similarity between the reference candidate region and other candidate regions in the respective Gabor response images. It finds the most similar candidate region among all the other corresponding regions, and then it performs a union operation for fusing the reference candidate region and the most similar one, which denotes flat safe regions for UAV landing.

Experimental results on finding regions for safe UAV landing with existing systems in the literature show that the proposed system outperforms the existing systems in terms of recall, precision and F-measure. In addition, we also simulate experiments to choose the best safe landing areas out of many safe landing zones given by the segmentation steps, according to emergency-situations by creating 500 random scenarios artificially, which includes a number of relative threats and more complex situations. Experimental results on classification through cost estimation show that the proposed segmentation approach is useful and works well for complex situations.

However, sometimes, the proposed system considers the region of water in an image as a false candidate region. This is due to the water surface appearing as a homogenous region for the proposed system. Therefore, we plan to introduce new features to integrate with the Gabor responses to improve the results under these scenarios in the near future. Also, during flight there is a high chance of capturing blurred images due to adverse climate effects and variations in height. In this case, the performance of the proposed system can degrade. One possible way to overcome this situation is that we can choose the option of traveling back to home base if necessary. Otherwise, we need to investigate new features that are robust to 
low contrast and poor quality images. These are all in the scope of improvements of the proposed work as part of future research.

\section{Acknowledgement}

This research work was supported by the Faculty of Computer Science and Information Technology, University of Malaya under a special allocation of Post Graduate Funds for the RP036B-15AET and PG063-2016 A project. This work was also supported by the Natural Science Foundation of China under Grant 61672273 and Grant 61832008, and the Science Foundation for Distinguished Young Scholars of Jiangsu under Grant BK20160021.

\section{References}

Aydin, M. and Kugi, E. (2016). Safe landing site detection using SRTM data for the unmanned aerial vehicles, In Proc. SIU, 2125-2128.

Aziz, S., Faheem, R, M., Bashir, M., Khalid, A., Yasin, A. (2016). Unmanned aerial vehicle emergency landing site identification system using machine vision. Journal of image and graphics, 4(1).

Fan, Z., Lu, J., Gong, M., Xie, H. and Goodman, E. D. (2018). Automatic tobacco plant detection in UAV images via deep neural networks, IEEE Journal of Selected Topics in Applied Earth Observations and Remote Sensing, Vol. 11, No. 3, 876-887.

Garcia-Pulido, J., Pajares, G., Dormido, S., de la Cruz, J. M. (2017). Recognition of a landing platform for unmanned aerial vehicles by using computer vision-based techniques, Expert Systems with Applications, 76, 152-165.

Garg, M., Kumar, A., Sujit, P. (2015) Terrain-based landing site selection and path planning for fixed-wing UAVs, In Unmanned aircraft systems (ICUAS), IEEE international conference on, 246-251.

Ghamisi, P., Couceiro, M. S., Benediktsson, J. A. and Ferreira, N. M. F. (2012). An efficient method for segmentation of images on fractional calculus and natural selection, Expert Systems with Applications, 39, 12407-12417.

Ghosh, A., Subudhi, B. N., Ghosh, S. (2012) Object detection from videos captured by moving camera by fuzzy edge incorporated Markov random field and local histogram matching. IEEE Transactions on Circuits and Systems for Video Technology, 22, 1127-1135.

Hinzmann, T., Stastny, T., Cadena, C., Siegwart, R and Gilitschenski, I. (2018). Free LSD: Prior-free visual landing site detection for autonomous planes. IEEE Transactions on Automation Letters, Vol. 3. No. 3. 2545-2552.

Huang, C. L., Chen, J. J., Chen, C. J. and Wu, Y. G. (2016). Geological segmentation on UAV aerial image using shape-based LSM with dominant color. In Proc. ICAINAW, 928-933.

Huang, L., Qu, H., Ji, P., Liu, X., Fan, Z. (2016). A novel coordinated path planning method using k-degree smoothing for multi-UAVs, Applied Soft Computing, 48, 182-192.

Kaff, A. A., Martin, D., Garcia, F., Escalera, A. D. La and Maria, J. (2018). Survey of computer vision and applications for unmanned aerial vehicles. Expert Systems with Applications, 92, 447-463.

Lange, S., Sünderhauf, N., Protzel, P. (2008). Autonomous landing for a multirotor UAV using vision. In International conference on simulation, modeling, and programming for autonomous robots (SIMPAR). 482-491.

Lee, J., Morton, Y. J., Lee, J., Moon, H.-S., \& Seo, J. (2017). Monitoring and Mitigation of Ionospheric Anomalies for GNSS-Based Safety Critical Systems: A review of up-to-date signal processing techniques. IEEE Signal Processing Magazine, 34, 96-110.

Li, X. (2013). A software scheme for UAV's safe landing area discovery. AASRI Procedia, 4, 230-235.

Mulatu, M. A. (2017). Energy cooperation in communication of energy harvesting tags, AEU-International Journal of Electronics and Communications, 71, 145-151. 
Patterson, T., McClean, S., Morrow, P., Parr, G., Luo, C. (2014). Timely autonomous identification of UAV safe landing zones. Image and Vision Computing. 32. 568-578.

Patterson, T., McClean, S., Parr, G., Morrow, P., Teacy, L., Nie, J. (2010) Integration of terrain image sensing with UAV safety management protocols. In International conference on sensor systems and software, springer. 36-51.

Saxena, A. K., Chaurasiya, V. K. (2018). Multi-resolution texture analysis for fingerprint based age-group estimation. Multimedia Tools and Applications, 77, 6051-6077.

Scherer, S., Chamberlain, L., Singh, S., (2012). Autonomous landing at unprepared sites by a full-scale helicopter, Robotics and Autonomous Systems, 60, 1545-1562. 